\title{
Vinculin Interacts with the Chlamydia Effector TarP Via a Tripartite Vinculin Binding Domain to Mediate Actin Recruitment and Assembly at the Plasma Membrane
}

\author{
Tristan R. Thwaites ${ }^{1,2}$, Antonio T. Pedrosa ${ }^{1,2,3}$, Thomas P. Peacock ${ }^{2}$ and \\ Rey A. Carabeo ${ }^{1,2,3 *}$ \\ ${ }^{1}$ Programme in Microbiology, Institute of Medical Sciences, University of Aberdeen, Aberdeen, UK, ${ }^{2}$ Medical Research \\ Council Centre for Molecular Bacteriology and Infection, Imperial College London, London, UK, ${ }^{3}$ School of Molecular \\ Biosciences, Washington State University, Pullman, WA, USA
}

\section{OPEN ACCESS}

Edited by:

Brice Rotureau,

Institut Pasteur, France

Reviewed by:

Agathe Subtil,

Institut Pasteur, France

Elizabeth Ann Rucks,

University of South Dakota, USA

Damien Paul Devos,

Centro Andaluz de Biología del

Desarrollo, Spain

${ }^{*}$ Correspondence:

Rey A. Carabeo

rcarabeo@vetmed.wsu.edu

Received: 20 October 2015 Accepted: 16 November 2015 Published: 30 November 2015

Citation:

Thwaites TR, Pedrosa AT, Peacock TP

and Carabeo RA (2015) Vinculin

Interacts with the Chlamydia Effector

TarP Via a Tripartite Vinculin Binding

Domain to Mediate Actin Recruitment and Assembly at the Plasma

Membrane

Front. Cell. Infect. Microbiol. 5:88. doi: 10.3389/fcimb.2015.00088
The mammalian protein vinculin is often a target of bacterial pathogens to subvert locally host cell actin dynamics. In Chlamydia infection, vinculin has been implicated in RNA interference screens, but the molecular basis for vinculin requirement has not been characterized. In this report, we show that vinculin was involved in the actin recruitment and F-actin assembly at the plasma membrane to facilitate invasion. Vinculin was recruited to the plasma membrane via its interaction with a specific tripartite motif within TarP that resembles the vinculin-binding domain (VBD) found in the Shigella invasion factor IpaA. The TarP-mediated plasma membrane recruitment of vinculin resulted in the localized recruitment of actin. In vitro pulldown assays for protein-protein interaction and imaging-based evaluation of recruitment to the plasma membrane demonstrated the essential role of the vinculin-binding site 1 (VBS1), and the dispensability of VBS2 and VBS3. As further support for the functionality of VBD-vinculin interaction, VBD-mediated actin recruitment required vinculin. Interestingly, while both vinculin and the focal adhesion kinase (FAK) colocalized at the sites of adhesion, the recruitment of one was independent of the other; and the actin recruitment function of the VBD/vinculin signaling axis was independent of the LD/FAK pathway.

Keywords: Chlamydia, signal transduction, vinculin, actin cytoskeleton, type III effectors

\section{INTRODUCTION}

In mammalian cells, the actin-binding protein vinculin associates with the cytoplasmic face of focal adhesions to facilitate linkage of integrin molecules to the actin cytoskeleton (Critchley et al., 1999). Structurally, vinculin is composed of three major domains: an $\mathrm{N}$-terminal head (Vh), a flexible proline-rich hinge region, and a C-terminal tail domain (Vt). Intramolecular associations between the head and tail domains constrain vinculin to an inactive state (Johnson and Craig, 1995). Vinculin activation requires the interaction with another focal adhesion protein, talin, which is mechanically stretched upon local application of tensile force at focal adhesions to expose vinculin binding sites (VBS) (Fillingham et al., 2005; Humphries et al., 2007; del Rio et al., 2009; Grashoff et al., 2010). Talin initially associates with Vh domain 1 (Vh1) of inactive vinculin through 
hydrophobic surface interactions (Gingras et al., 2005). Switching to an open active conformation allows for complete binding of vinculin to talin (Case et al., 2015). In its active form, vinculin is capable of direct interaction with Arp2/3, actin, phosphatidylinositol (4,5)-bisphosphate (PIP2), and paxillin (Turner et al., 1990; Hüttelmaier et al., 1998; DeMali et al., 2002). Thus, the binding of vinculin to talin can facilitate a localized increase in the number actin filaments and the maturation of focal adhesions.

Unsurprisingly, a number of bacterial pathogens have been shown to employ vinculin to facilitate infection of host cells and dissemination within tissues. Focal adhesion proteins including talin and vinculin have been found to accumulate within Salmonella-induced membrane ruffles (Finlay et al., 1991). More recently, the Rickettsia cell surface antigen, Sca4, and the Shigella flexneri effector protein, IpaA, have been shown to bind and activate vinculin (Tran Van Nhieu et al., 1997; Tran Van Nhieu and Izard, 2007; Park et al., 2011a). Both Sca4 and IpaA connect to host F-actin by functioning as talin mimics to bind and activate vinculin (Hamiaux et al., 2006; Park et al., 2011a,b). In each case, the virulence factors harbor at least two VBSs.

Signaling required for Chlamydia invasion is initiated by the attachment of the infectious elementary body (EB) to the host cell surface, where it either engages a number of host cell receptors and/or activate its type III secretion system (T3SS) to translocate the effector TarP (Clifton et al., 2005; Elwell et al., 2008; Lane et al., 2008; Mehlitz and Rudel, 2013). It is unclear if an individual EB simultaneously activates all of the pathways experimentally implicated or if the infecting organism display preference for one specific pathway depending on the context of infection, including cell type and two- or three-dimensional culture configuration. In addition, the functional relationships between the characterized pathways have not been fully elucidated. Regardless, the net result is the robust recruitment of actin at the sites of chlamydial adhesion, leading to the engulfment and internalization of the EB (Carabeo et al., 2002; Subtil et al., 2004; Balañá et al., 2005).

TarP is a chlamydial virulence factor that functions to recruit signaling molecules at the plasma membrane via a number of domains that resemble mammalian signaling motifs (Lane et al., 2008; Carabeo, 2011). We have previously identified the signaling function of the $\mathrm{N}$-terminal phosphodomain of TarP of Chlamydia trachomatis by interacting with Src homology 3 (SH3)-domain containing adapter proteins to recruit two forms of Rac GTPases to induce an Arp2/3 complex-mediated nucleation of actin polymerization at the sites of invasion (Lane et al., 2008). Also, we have recently reported on the role in actin recruitment of the FAK-binding motif (LD) present in TarP (Thwaites et al., 2014). Jewett et al. also functionally characterized the role of the TarP actin-binding domains in actin nucleation (Jewett et al., 2006). The multiple mechanisms of actin recruitment and polymerization beg the question of functional interaction. Jewett et al. demonstrated a cooperation between Arp2/3- and direct TarP-mediated actin nucleation processes (Jiwani et al., 2012).

To date, the question of whether vinculin plays a role in Chlamydia invasion remains largely unknown, but RNA interference screens have identified vinculin being necessary for C. trachomatis L2 infection in vitro (Elwell et al., 2008;
Gurumurthy et al., 2010). Both screens assessed inclusion formation, and not chlamydial invasion specifically. In this study, we show that vinculin is necessary for invasion, and is recruited by Chlamydia at the sites of adhesion in a FAK-independent, but TarP-dependent fashion. Three putative VBSs located at the C-terminus of TarP of Chlamydia caviae comprising the vinculin binding domain (VBD) was functionally characterized. Examination of other TarP orthologs revealed conservation amongst the chlamydial species, underscoring its biological importance. VBD was the sole domain in TarP that interacted with vinculin, and the each VBS displayed varying levels of vinculin binding in vitro. The VBD motif is competent in signaling to mediate localized actin recruitment at the plasma membrane in a vinculin-dependent, but FAKindependent manner. Furthermore, the presence of the proximal FAK-binding LD motif was not required. Enhancement in the frequency of actin recruitment was not observed in the presence of the LD motif.

\section{MATERIALS AND METHODS}

\section{Reagents, Cell Lines, and Organisms}

Anti-HA.11 clone 16B12 mAb was purchased from Covance; anti-FAK (phospho Y397) pAb and anti-vinculin mAb were from Abcam. Anti-rabbit or anti-mouse IgG secondary antibodies, either Alexa Fluor 488 or 594 were purchased from Invitrogen. Phalloidin conjugated to Alexa Fluor dye was purchased from Invitrogen. Cos7 (ATCC CRL-1651) and HeLa 229 (ATCC CCL-2.1) were routinely grown in DMEM supplemented with $10 \%$ FBS, $2 \mathrm{mM}$ L-glutamine, and $10 \mu \mathrm{g} / \mathrm{ml}$ gentamicin. Subcultivation was at 1:4 ratio. The cells were used at passage $<15$. $\mathrm{FAK}^{-/-}$mouse embryo fibroblasts (MEFs; CRL2644) and matched $\mathrm{FAK}^{+/+}$cells (CRL-2645) were purchased from LGC standards. $v \mathrm{l}^{-/-}$and matched $v \mathrm{l}^{+/+} \mathrm{MEFs}$ (Marg et al., 2010) were generously provided by Dr. Wolfgang Ziegler (Hannover Medial School). All MEFs were cultured in DMEM $+10 \%$ FBS and subcultured at 1:4 ratio. Cultured cells were grown in a humidified $5 \% \mathrm{CO} 2$ incubator at $37^{\circ} \mathrm{C}$. C. caviae strain GPIC was propagated in HeLa cells grown in DMEM $+10 \%$ FBS supplemented with gentamicin $(10 \mu \mathrm{g} / \mathrm{ml})$. Harvest of elementary bodies was by discontinuous density gradient centrifugation in Renografin (Bracco Diagnostics), as previously described (Caldwell et al., 1981).

\section{Cloning}

The VBD (VBS1-LLEAARNTTTMLSKTLSKV; Thr $^{714}-\mathrm{Ser}^{880}$ ), $\mathrm{VBS1}^{\text {mut }}$-2-3 (Mutant VBS1-LLESSRNTTTMSSSKTSSSSV; Thr $^{714}$ $\left.\mathrm{Ser}^{880}\right), \Delta$ VBS1 $\left(\mathrm{Thr}^{714}\right.$-Arg $\left.{ }^{832}\right), \Delta$ VBS2,3 $\left(\right.$ Leu $\left.^{837}-\mathrm{Ser}^{880}\right)$, $\triangle$ VBS1,2 $\left(\right.$ Thr $^{714}-$ Thr $\left.^{784}\right)$, the LD $\left(S^{6} r^{640}-\right.$ Pro $\left.^{740}\right)$, and the LDVBD (Ser ${ }^{640}-$ Ser $^{880}$ ) fragments were amplified from the C. caviae GPIC TarP clone (Clifton et al., 2005) using primer pairs indicated in Table 1. Primers were engineered with KpnI linkers. GPIC TarP deleted of the proline rich domain (PRD; $\mathrm{Ile}^{355}$ $\mathrm{Val}^{399}$ ) to aid solubility during overexpression was generated by inverse PCR from a GPIC TarP clone (Clifton et al., 2005) using primer pair 13-14. Deletion of the VBS3 $\left(\mathrm{Leu}^{746}-\mathrm{Ala}^{764}\right)$ or VBS2 $\left(\mathrm{IIe}^{806}-\mathrm{IIe}^{824}\right)$ was also facilitated by inverse PCR using primer 
TABLE 1 | Primers used for PCR amplification in this study.

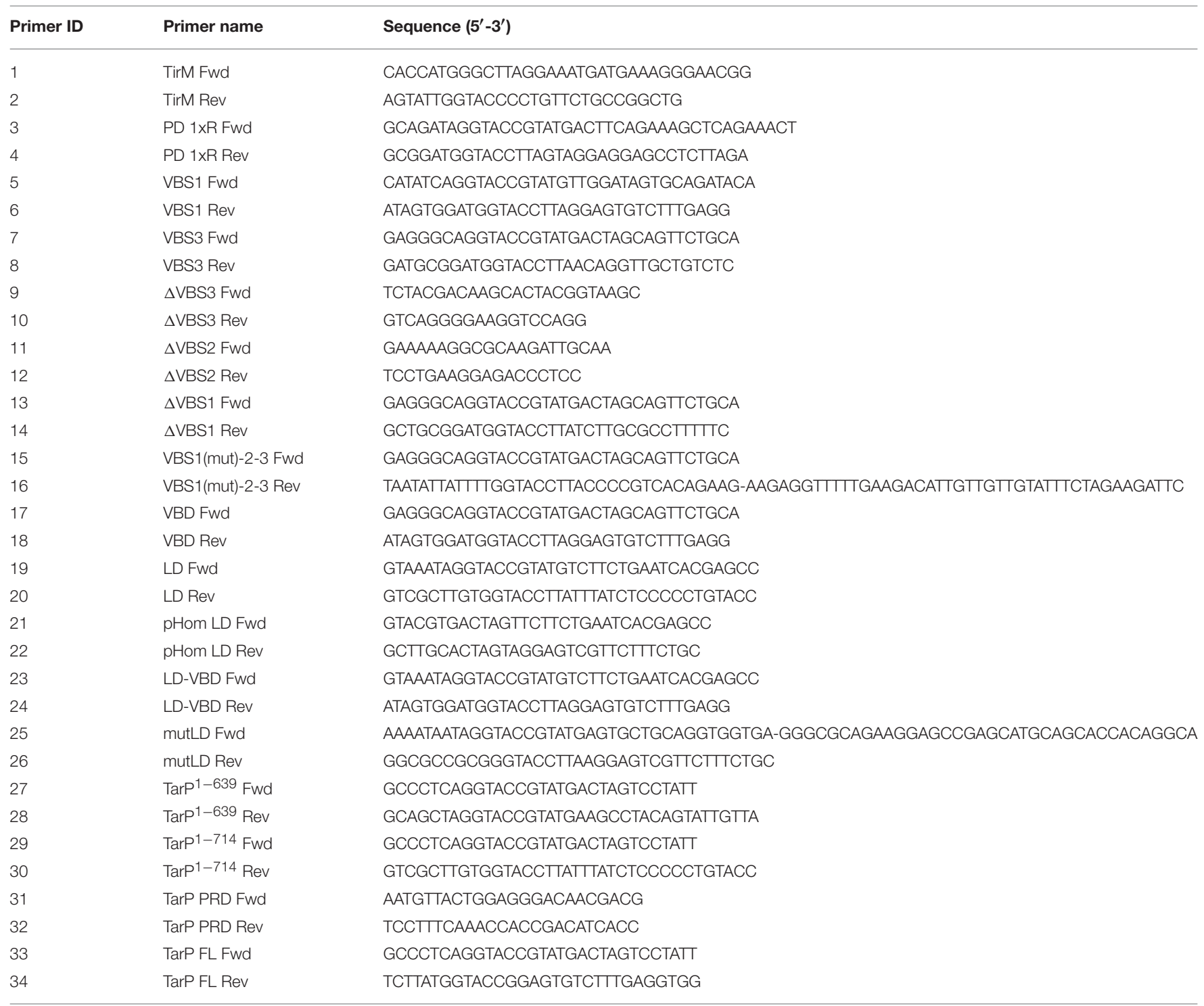

pairs $31-32,9-10$ and $11-12$, respectively. The TarP deletion variant $\left(\mathrm{Met}^{1}-\mathrm{Thr}^{714}\right)$ was generated from GPIC TarP clones $(\triangle \mathrm{PRD})$ using primer pairs $9-10$ and $11-12$ engineered with Kpn1 linkers for fusion with the TirM derivative of the TirMC plasmid (pKC87). This plasmid has been previously described by Campellone et al. (2004) and was generously provided by Professor John Leong (Tufts University). Construction of TirM involved PCR amplification of part of TirMC that contained amino acids 1-66, which encodes the essential membrane targeting domain of the Newcastle Disease Virus HN surface protein, the hemagglutinin (HA) epitope tag and Tir amino acids 260-395. The "C" region of TirMC was excluded, and in its place a unique Kpn 1 site was introduced at the 3 ' end of the TirM openreading frame using primer pair $1-2$. This facilitated the cloning of TarP fragments to create TirM-TarP fusion derivatives. Bluntend TirM PCR products were cloned directionally by TOPO ${ }^{\circledR}$ Cloning into a $\mathrm{pENTR}^{\mathrm{TM}} / \mathrm{D}-\mathrm{TOPO}^{\circledR}$ entry vector (Invitrogen) to create pENTR-TirM. The products generated for TarP-FL, its deletion variants, and isolated regions of interest were digested with KpnI and subcloned into linearized pENTR-TirM to generate translational fusions with TirM at the N-terminus. Expression clones were generated through LR recombination between entry clone and pcDNA-Dest40 (Invitrogen), which had the CMV promoter to drive expression in mammalian cells. All constructs were verified by DNA sequencing.

\section{Transfection and Indirect Confocal Microscopy}

Cos7 were transfected with full-length TarP, the VBD, VBD derivatives, the LD, or the LD-VBD using Lipofectamine 2000 (Invitrogen) as described by the manufacturer. At $32 \mathrm{~h}$ posttransfection, the cells were fixed by adding $4 \%$ paraformaldehyde. Antibodies diluted in $1 \times$ PBS to their respective working concentrations (anti-HA.11 clone 16B12 1:500; anti-vinculin 
1:250) were added to fixed cells and incubated $37^{\circ} \mathrm{C}$ for $1 \mathrm{~h}$. Actin was detected with phalloidin (1:250 dilution) conjugated to Alexa Fluor dye. Coverslips were mounted onto glass slides using mowiol. Sample visualization was performed at RT on a laser scanning microscope (LSM 510; Carl Zeiss) using an oil-immersion PlanApochromat $63 \times / 1.40 \mathrm{NA}$ differential interference contrast. Images were processed using NIH ImageJ freeware (Schneider et al., 2012) or Adode Photoshop CS5. Pixel intensities were measured using ImageJ freeware. Incidences of colocalization were assessed visually in a single-blind experiment by someone not involved with image acquisition. For the generation of heat maps of pixel intensity, images for each cell population were taken with identical exposure times and on the same day to allow accurate comparison of fluorescence intensities between images. Images were exported into NIH ImageJ for analysis.

\section{Co-Immunoprecipitation and Western Blot Analysis}

Briefly, Cos7 cells from five 6-well culture plates expressing HAtagged proteins were washed once with phosphate-buffered saline (PBS) at room temperature and then lysed with $0.5 \mathrm{ml}$ ice-cold IP lysis buffer (Pierce). Cells were scraped, transferred into a $1.5 \mathrm{ml}$ microcentrifuge, and vortexed briefly. Lysis was continued on ice for $10 \mathrm{~min}$, after which the cell debris was pelleted at $15,000 \mathrm{rpm}$. in a microcentrifuge. Lysates were incubated with anti-HA magnetic beads (Pierce) at $4{ }^{\circ} \mathrm{C}$ with rocking as described by the manufacturer. The beads were then washed twice with $0.3 \mathrm{ml} 0.05 \%$ TBS-T (Tris-buffered saline supplemented with Tween) and then eluted with two $50-\mu \mathrm{l}$ washes of $2 \mathrm{mg} / \mathrm{ml} \mathrm{HA}$ peptide (Pierce) in TBS. The eluted samples were mixed with Laemmli buffer and boiled prior to gel electrophoresis. Proteins were probed with anti-vinculin (1:1000 dilution) (Abcam) or anti-HA (1:1000 dilution) (Covance) antibodies followed by the addition of HRP-conjugated secondary antibody at 1:1000 dilution (Cell Signaling Technology).

\section{Bacterial Infection}

For EPEC, bacterial cultures were primed for infection as previously described (Wong et al., 2012) with the following modification. A 1:500 dilution of overnight culture was used to prime the bacterial culture for infection. Cos7, $\mathrm{ccl}^{+/+}$and $\mathrm{cl}^{-/-}$ cells were grown in 24 -well cell culture plates to $80 \%$ confluence. The cells were transfected with $100 \mathrm{ng}$ plasmid DNA. Transfected cells were incubated at $37^{\circ} \mathrm{C}$ in a humidified incubator for $32 \mathrm{~h}$ prior to infection. Cells were infected as previously described (Campellone et al., 2004). The infection was allowed to proceed for $4 \mathrm{~h}$ in the presence of gentamicin $(200 \mathrm{mg} / \mathrm{ml})$ (Gibco) after the first hour. $\triangle$ tir EPEC were visualized using DAPI diluted at 1:1000. For Chlamydia, HeLa, $\mathrm{vcl}^{-/-}, \mathrm{vcl}^{+/+}, \mathrm{FAK}^{-/-}$, and $\mathrm{FAK}^{+/+}$cells were infected as previously described (Thwaites et al., 2014) with the following modifications. Cells were infected at a multiplicity of infection (MOI) of 50 for C. caviae GPIC and spun (1500 rpm for $3 \mathrm{~min}$ ) at $4^{\circ} \mathrm{C}$ ( HeLa) or room temperature (RT; $v l^{-/-}, v l^{+/+}, \mathrm{FAK}^{-/-}$and $\mathrm{FAK}^{+/+}$) to allow maximum chlamydial adherence to target cells.

\section{Invasion Assay}

Efficiency of C. caviae strain GPIC invasion was performed using a double staining and fixation protocol as described previously (Carabeo et al., 2002; Subtil et al., 2004) with the following modifications. $v \mathrm{cl}^{-/-}$and $v \mathrm{cl}^{+/+}$cells were infected C. caviae at MOI of 1 and spun at $1500 \mathrm{rpm}$ for $3 \mathrm{~min}$ at RT to synchronize infection. Unattached EBs were removed by washing cells three times with room temperature (RT) IMDM media. Pre-warmed IMDM media was added and the infection was allowed to proceed at $37^{\circ} \mathrm{C}$ for the appropriate time p.i. Coverslips were washed three times in PBS and fixed with $4 \%$ paraformaldehyde for 15 min. Extracellular EBs were labeled with a C. trachomatis anti-LPS 1:750 (Abcam) primary and Alexa Fluor 488 conjugated secondary antibody (Invitrogen). Cells were permeabilised with $0.25 \%$ Triton X-100 for $5 \mathrm{~min}$ and washed $3 \times$ with PBS before a secondary fixation step with $4 \%$ PFA for 15 min. Total EBs were labeled with an anti-Chlamydia antibody as the primary antibody at 1:750 dilution (Abcam) and Alexa Fluor 594-conjugated secondary antibody (Invitrogen).

\section{Bioinformatics}

All figures involving the alignment or phylogenetic analysis of nucleic acid sequence were generated using ClustalW. Parameters for alignments shown are indicated within the figure legends describing the alignment. Helical wheel representations were generated using PROTEAN from DNASTAR inc.

\section{Statistical Analysis}

All statistical analyses were run using the program StatPlus. Unless stated otherwise, statistical differences were tested using One-way analysis of variance (ANOVA) and Tukey's post-hoc test. Box and Whisker Plots show range (statistical outliers excluded), the lower and upper quartile values (box) with median (horizontal line) and mean (diamond). Whiskers extend from each end of the box to the 10th and 90th percentiles. Where stated, the significance level $\alpha$ was set to 0.05 or 0.01 . All $p$ values $\leq \alpha$ were considered statistically significant.

\section{RESULTS}

\section{Chlamydia Requires Vinculin for Invasion}

Vinculin has been shown to be a target of a number of microbial pathogens as they take advantage of its ability to mobilize actin. Given previous findings demonstrating the involvement of various focal adhesion components during Chlamydial infection (Coombes and Mahony, 2002; Elwell et al., 2008; Gurumurthy et al., 2010), we sought to determine in greater detail the involvement of vinculin in Chlamydia invasion. Using HeLa cells, vinculin recruitment to the sites of invasion was evaluated. HeLa cells were infected with C. caviae GPIC and vinculin recruitment monitored at $0,10,30,60,90$, and 120 min after synchronization by temperature-shift to $37^{\circ} \mathrm{C}$. Cells were immediately fixed with freshly prepared $4 \%$ paraformaldehyde at the designated time points. As shown in Figure 1A, vinculin was observed to be strongly concentrated around EBs as early as $10 \mathrm{~min}$ postinfection (p.i.). From confocal microscopy images of infected cell 


\section{$\%$ of vinculin positive EBs}

A
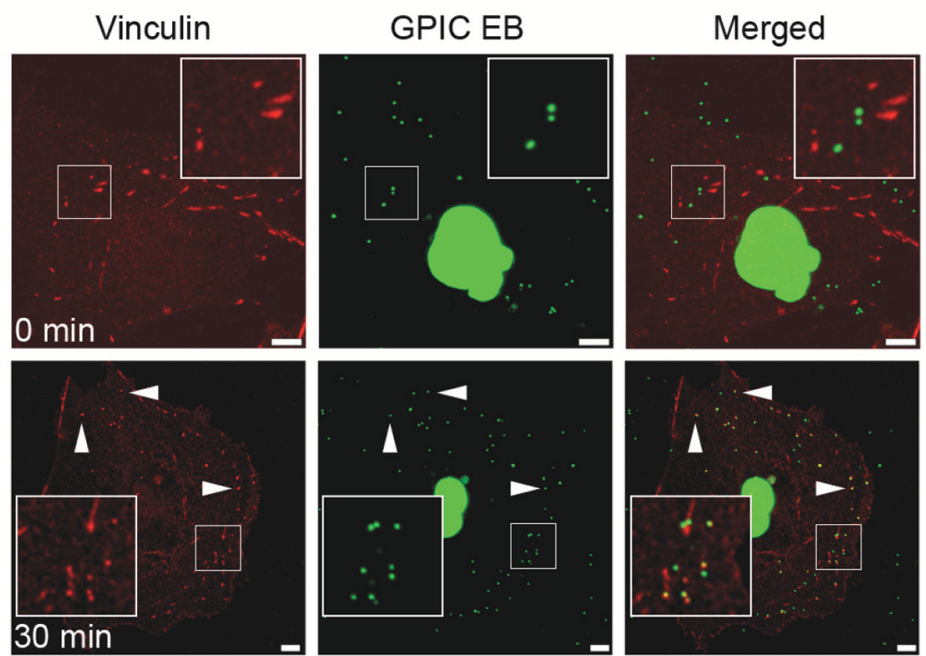

B

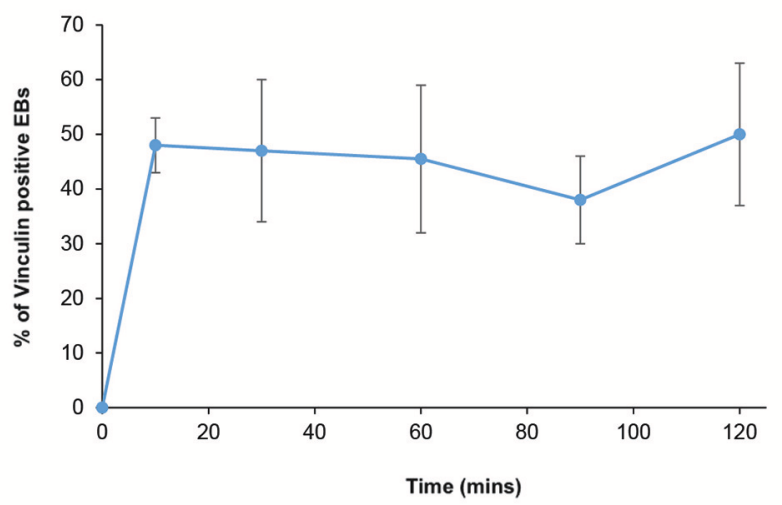

C

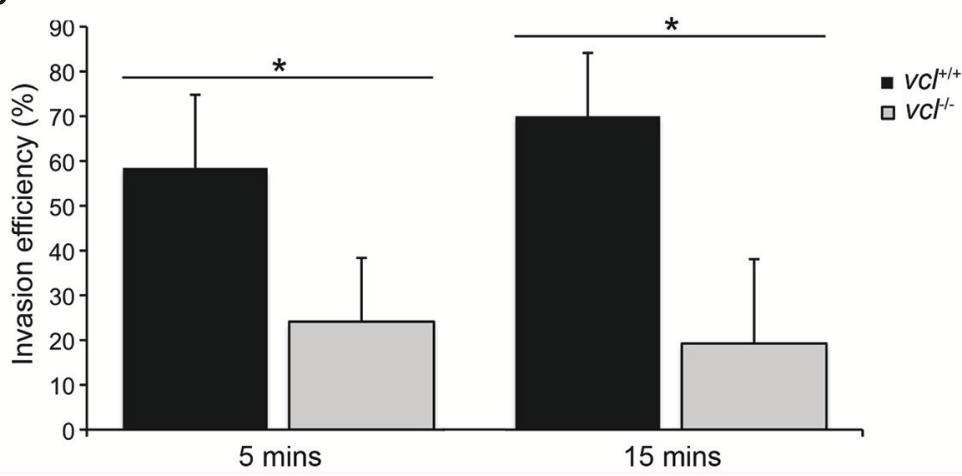

FIGURE 1 | Chlamydia recruits vinculin to sites of invasion. (A) C. caviae (GPIC) elementary bodies (EBs) were added to Cos 7 cells at $4^{\circ} \mathrm{C}$, and shifted to $37^{\circ} \mathrm{C}$ by the addition of pre-warmed media to synchronize invasion. The infection was allowed to proceed for 0, 10, 30, 60, 90, or 120 min at which time the cells were fixed and process for immunofluorescence staining and microscopy. Cells were stained with an anti-vinculin antibody (red) and Chlamydiae were visualized by DAPI (green). Note the increase in the number of co-localization events from 0 to $30 \mathrm{~min}$. Images for the other time points are shown in Figure S1. Scale bars: $5 \mu \mathrm{m}$. White arrowheads indicate vinculin colocalizing with GPIC EBs. (B) Colocalization frequency per cell was calculated for each time point up to 120 min post-infection, with at least 50 cells analyzed per time point. There is a rapid increase of vinculin recruitment by $10 \mathrm{~min} \mathrm{p.i.,} \mathrm{which} \mathrm{was} \mathrm{sustained} \mathrm{up} \mathrm{to} 120 \mathrm{~min} \mathrm{p.i.} \mathrm{(C)} v \mathrm{I}^{+/ /+}$and $v \mathrm{Cl}^{-/-}$ cells were infected with C. caviae (GPIC). Infection was allowed to proceed up to $15 \mathrm{~min}$ post-temperature shift. Cells were fixed and processed for the invasion assay as detailed in the Materials and Methods Section. GPIC invasion was evaluated in $\mathrm{cCl}^{+/+}$and $\mathrm{vCl}^{-/-}$cells. $\mathrm{vCl}^{-/-}$cells were less able than wild-type to support GPIC invasion at 5 or 15 min post-infection (p.i.). Data are from a minimum of 150 cells from three independent experiments, and expressed as means \pm SD. Asterisk and bar indicates statistical significance between specific groups (One-way ANOVA, Tukey's post-hoc test, $P<0.008$ ). 
monolayers, we quantified the levels of colocalization of EBs to vinculin. The graph in Figure $1 \mathbf{B}$ shows recruitment at $10 \mathrm{~min}$ p.i., and that the steady state levels remained elevated until at least 120 min p.i.

To address the chlamydial requirement for vinculin, we exploited mouse embryo fibroblasts (MEFs) ablated for the $v c l$ gene $\left(\mathrm{vcl}^{-/-}\right)$. To avoid functional redundancy in signaling to the actin remodeling machinery, C. caviae GPIC was chosen because it lacked the $\mathrm{N}$-terminal phosphodomain previously shown to promote Sos1- and Vav2-mediated actin recruitment in C. trachomatis (Lane et al., 2008). The absence of the phosphodomain in the C. caviae TarP ortholog should enable us to definitively assign signaling pathways to the motifs in the C-terminal half of TarP. Invasion assays in wild type (WT) and $\mathrm{vcl}^{-/-}$MEFs by C. caviae GPIC EBs were performed and quantified as previously described (Thwaites et al., 2014). Invasion was monitored at 5 and 15 min post-infection (p.i.), and data was expressed as invasion efficiency per cell. As shown in Figure 1C, the invasion efficiencies of $\mathrm{vcl}^{-/-}$MEFs were significantly different from the $\mathrm{vcl}^{+/+}$MEFs at both time points post infection (p.i.) $(p<0.005)$. The incomplete inhibition of invasion in vinculin-depleted cells was likely to be related to redundant invasion pathways, including the FAK-dependent pathway that signals from the LD domain of TarP (Thwaites et al., 2014). Overall, the highly localized recruitment of vinculin to the sites of entry, and the requirement for efficient invasion suggest that vinculin has an essential role in the infection of cultured cells by C. caviae GPIC.

\section{Identification of the Vinculin Binding Domain in the Chlamydial Effector TarP}

Some proteins that interact with vinculin, including the focal adhesion-associated talin, require VBS consisting of a 19-residue consensus motif (LLxAAKAVADAxSKLLKAx). This consensus sequence was determined from the alignment of the $11 \mathrm{VBS}$ of talin (Gingras et al., 2005), the three VBSs of IpaA (Park et al., 2011b) and the two VBSs of sca4 (Park et al., 2011a). We hypothesized that the robust vinculin recruitment may involve the virulence factor TarP due to its established function as a signaling scaffold (Lane et al., 2008). Through bioinformatics analyses, candidate VBS motifs were identified in the TarP orthologs of all chlamydial species investigated (Figure 2A). Together, these VBSs comprised the VBD, with VBS1 being the most C-terminal (Figure 2B).

\section{C. caviae TarP is Sufficient to Recruit Vinculin}

We tested the putative TarP VBD for its ability to recruit vinculin to the plasma membrane using a previously described assay based on the clustering of ectopically expressed and plasma membranetargeted TarP fused to the extracellular domain of the Tir protein of enteropathogenic Escherichia coli (EPEC) (Thwaites et al., 2014). Briefly, the TarP domain of interest (Figure 3A) was fused to the extracellular and transmembrane domains of Tir. When transiently expressed in host cells, the Tir-TarP fusions (TirMTarP) were targeted to the plasma membrane where they are clustered by adhered $\triangle$ tir EPEC to initiate signaling (Campellone et al., 2004; Thwaites et al., 2014). Through deletion analysis of TarP, the heterologous EPEC-based system confirmed the role of VBD (TirM-TarP ${ }^{714-880}$ ) in vinculin recruitment. In Figure 3B, vinculin recruitment was induced by full-length TarP (TirMTarP FL). Removing the C-terminal 166 amino acid residues led to the loss of vinculin recruitment, indicating that this region fully accounted for the vinculin recruitment activity of full-length TarP. Scoring individual EPEC particles for colocalization with vinculin and expressing data as recruitment frequency revealed an increase of three-fold for TarP-FL relative to TirM-Tar $\mathrm{P}^{1-714}$ (Figure 3C; $p<0.005$; ANOVA and Tukey-Kramer post-hoc test). Taken together, we concluded that TarP itself could recruit vinculin, and we attribute this activity to the putative VBD motif located within the last 166 amino acids.

Interestingly, vinculin was not recruited by the FAKinteracting LD domain present in TirM-TarP ${ }^{1-714}$, despite the known role of this protein in the FAK signaling pathway in focal adhesions. To confirm this observation, TirM-VBD and TirM-LD (Figure 4A) were evaluated for vinculin recruitment in transiently transfected Cos7 cells. Fusion proteins localized to the plasma membrane were clustered by EPEC $\Delta$ tir mutants adhered to the cell surface. After $1 \mathrm{~h}$, the samples were fixed for staining with antibody to vinculin. Images were acquired and the frequency of vinculin recruitment was determined for each sample. In Figure 4B, there was a noticeably decreased incidence of vinculin recruitment in the TirM-LD sample compared to TirM-VBD. Quantification of recruitment incidence revealed that TirM-VBD was statistically significant different from the negative control TirM, in contrast to TirM-LD.

\section{The VBD Domain Requires Vinculin to Recruit Actin}

Having established the role of VBD in vinculin recruitment, we next addressed the functionality of this interaction in regards to actin recruitment and filamentous actin (F-actin) assembly. The ability of the VBD domain to recruit actin was compared to the LD motif, which was previously shown to induce a FAK-dependent signaling to mediate actin recruitment, and thus was the positive control. Using the EPEC-based assay, plasma membrane-localized TirM-VBD, TirM-LD, or TirM was clustered by the adhered EPEC to induce signaling. Recruitment of actin and vinculin was assessed for each sample by staining with phalloidin and anti-vinculin antibody, respectively. As shown in Figure 5A, TirM-VBD and TirM-LD were able to recruit actin, but only the former efficiently localized vinculin underneath the adhered EPEC bacteria. The negative control TirM recruited neither actin nor vinculin. Incidence of actin recruitment was quantified for each sample (Figure 5B). Incidences for both TirM-VBD andTirM-LD were statistically significantly different than that for the negative control. Based on these data, we conclude that the VBD domain is competent in signaling to recruit actin.

We tested directly the dependence of VBD-mediated actin recruitment on vinculin using $\mathrm{vcl}^{-/-}$MEFs. Using the same EPEC-based assay in conjunction with TirM-VBD, the transfected fibroblasts were evaluated for actin recruitment 
A

L2 VBS2: 938-DKGN L F Q A A A V T Q A L G N V A G K V N LAIQG-967

L2 VBS1: 981-VGRD L

GPIC VBS3: 745-GSPD \begin{tabular}{llllllllllllllllllll} 
L & H & $G$ & A & A & K & G & V & A & D & S & L & S & N & L & L & Q & A & A & TSPTT-7 \\
\hline
\end{tabular}

GPIC VBS2: 805-SPSG I P G A A A N V T A T L S S V A

GPIC VBS1: 849-QGKQ $\begin{array}{llllllllllllllllllll}\text { L } & \text { L } & \text { E } & \text { A } & \text { A } & \text { R } & \text { N } & \text { T } & \text { T } & \text { T } & \text { M } & \text { L } & \text { S } & \text { K } & \text { T } & \text { L } & \text { S } & \text { K } & \text { V } & \text { TGSPP-873 }\end{array}$

CAb VBS 3: 735-EAPD L

CAb VBS2: 794-APSG I P

CAb VBS 3: 839-QRKQ L A D A A

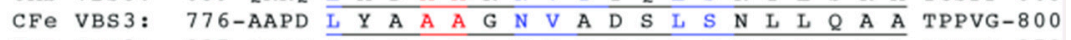

CFe VBS 2: 835-SPSG I P

CFe VBS1: $\quad 878-Q G Q K$ L

MOPN VBS2: 943-EKVS L \begin{tabular}{llllllllllllllllllll}
\hline & D & A & A & $K$ & $N$ & V & T & $Q$ & A & L & T & S & V & T & N & K & V & TLAMQ-967
\end{tabular}

MOPN VBS1: 987-IGQN L

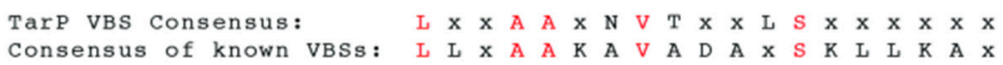

B

c. trachomatis L2

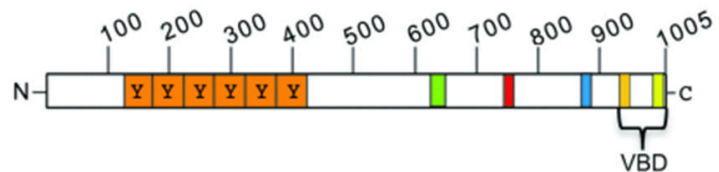

c. caviae

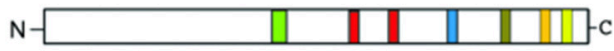

C. abortus

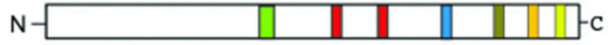

C. felis

c. muridarum
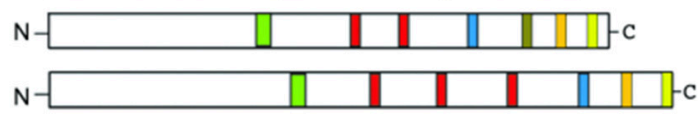

\begin{tabular}{|l|l|l|l|}
\hline $\mathrm{Y}$ & $\mathrm{Y}$ & $\mathrm{Y}$ & Tyrosine-rich repeat region \\
\hline
\end{tabular}

Proline rich domain

Actin binding domain

【 Lo domain

【 vBS1 IVBS2 IVBS3

FIGURE 2 | TarP orthologs harbor multiple vinculin binding sites. (A) Schematic of TarP orthologs from C. trachomatis serovar L2 (L2), C. trachomatis serovar D (D), C. trachomatis serovar A (A), C. caviae (GPIC), C. abortus (Cab), C. felis (CFe), and C. muridarum (MoPN). Indicated are the locations of the tyrosine-rich phosphorylation domain (orange box), the proline rich domain (green box), actin binding domains (red box), and the LD domain (blue box). Vinculin Binding Site 3 (VBS3; brown box), VBS2 (light orange box), and C-terminal VBS1 (yellow box) together comprise the Vinculin Binding Domain (VBD). (B) ClustalW sequence alignment of the putative VBS motifs. The numbers indicate the amino acid residue of the amino terminus or carboxy terminus. The consensus sequences shown are based on homology greater than 50\%. A 19-residue consensus motif (LLXAAKAVADAxSKLLKAx) was generated by aligning by aligning the 11 VBSs of talin, the three VBSs of IpaA, and the two VBSs of sca4 for comparison to the TarP LD consensus ( $x$ xxAAxNVTxxLSxxxxxx). Identical amino acids are in red. Similar residues are in blue. "x" indicates any amino acid.

at the base of the adhered EPEC. The contrasting cell morphologies of $\mathrm{vcl}^{+/+}$and $\mathrm{vcl}^{-/-}$MEFs were expected and consistent with previous reports. Analysis of the confocal images revealed the essential role of vinculin in VBD-mediated actin recruitment (Figure 6A). Punctate F-actin aggregates could be observed below the adhered EPEC in WT MEFs. In contrast diffuse phalloidin staining were seen in the knockout cells. Quantification of the incidence of actin recruitment confirmed the role of vinculin. The frequencies of actin recruitment were significantly greater $\left(>2.5\right.$-fold) relative to the $\mathrm{vcl}^{-/-}$samples (Figure 6B; $p<0.005$; ANOVA and Tukey-Kramer post-hoc test).

\section{Functional Analysis of VBD Deletion Derivatives Reveals an Important Role for VBS1 in Vinculin Recruitment}

The S. flexneri effector IpaA possesses three functional VBSs in tandem arrangement, of which the highest affinity C-terminal motif functioning as the trigger for vinculin activation (Tran
Van Nhieu et al., 1997). Like the IpaA effector, C. caviae TarP harbors three VBS motifs. Helical wheel representations of the C. caviae TarP VBS motifs showed VBS1 to be most similar to the C-terminal VBS of IpaA (IpaA-VBS1), with comparable directions and magnitudes of the hydrophobic moments. Conversely, VBS2 or VBS3 have smaller magnitudes of the hydrophobic moments, indicating lower amphiphilicity (Figure S2). Because of the functional differences between the IpaA VBSs, we investigated if the same could be true for TarP VSB1, 2, and 3. Various combinations of VBSs were investigated for vinculin recruitment frequency in the context of the EPEC system (Figure 7A). As shown in Figures 7B,C, deletion of either VBS2 or VBS3 while retaining VBS1 (TirM- $\triangle$ VBS2, TirM- $\Delta$ VBS3, or TirM- $\Delta$ VBS2,3) had minimal influence on the incidence of vinculin recruitment in comparison to the WT VBD, hinting at the greater functional importance of VBS1. This was confirmed through the progressive deletions of the VBD that removed either VBS1 only or VBS1 and VBS2, with both deletion constructs leading to notable decreases in the 
A

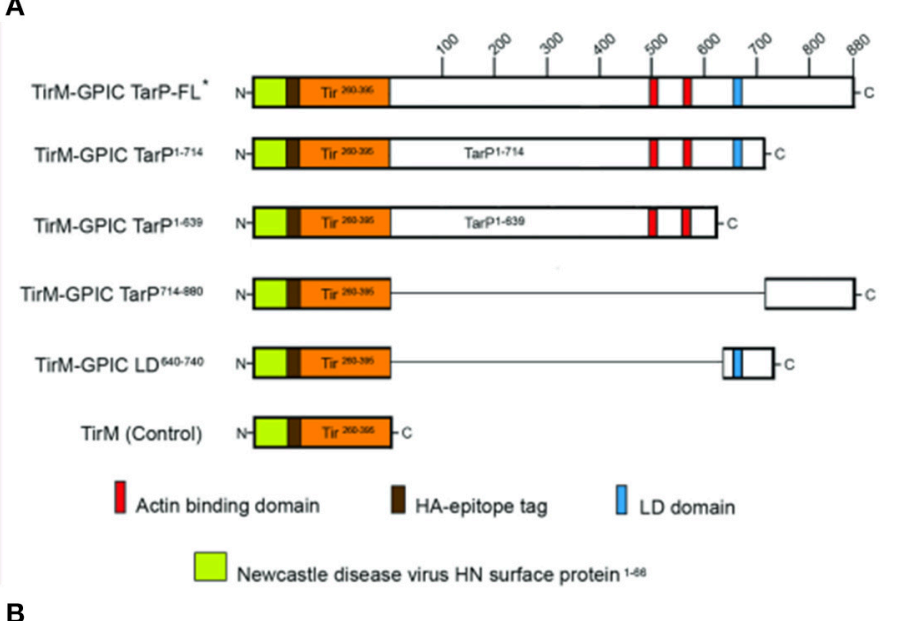

B
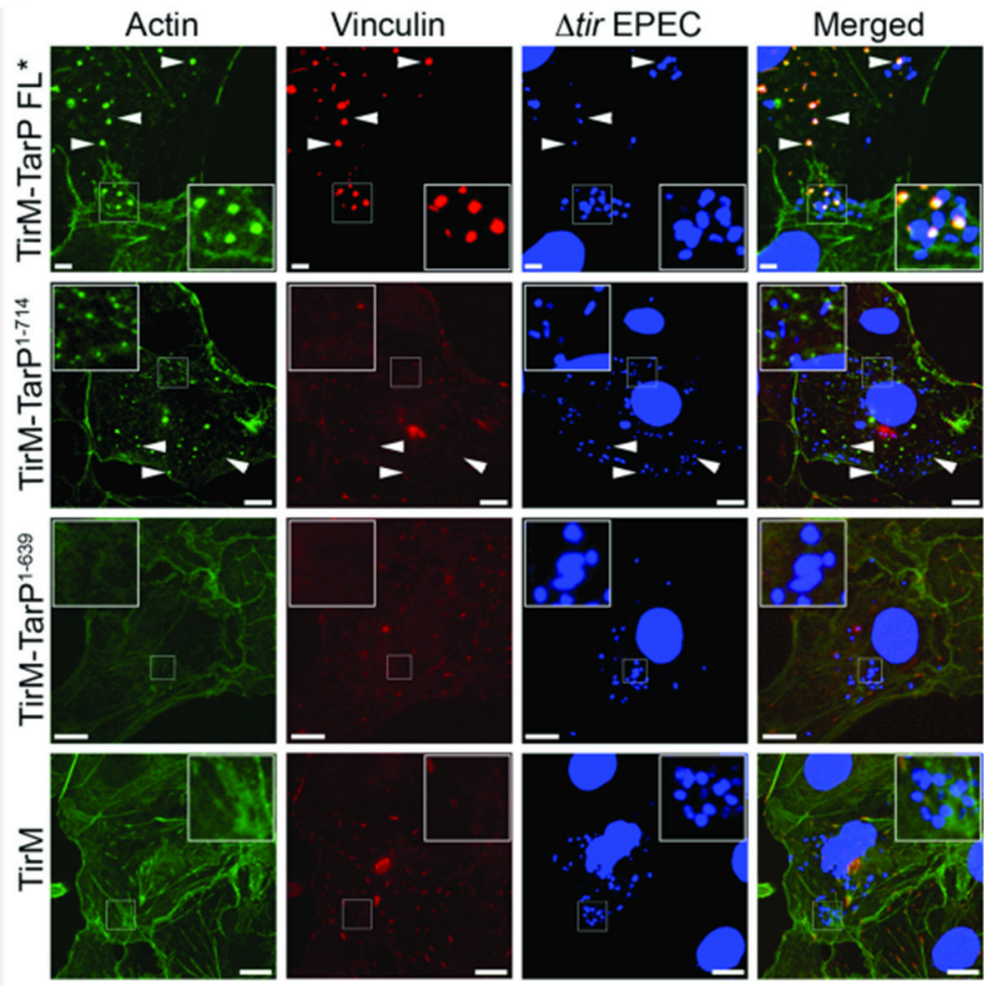

C

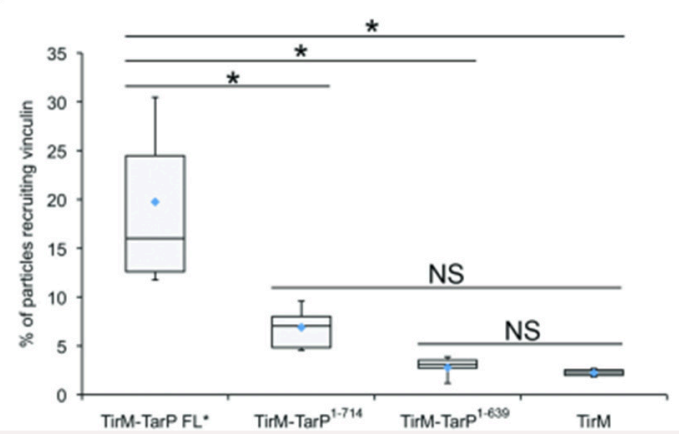

FIGURE 3 | C. caviae TarP-mediated vinculin recruitment requires the vinculin binding domain. (A) Schematic indicating the locations of the membrane targeting sequence (yellow box), Ha-tag (brown box), TirM (amino acids 260-395; orange box), actin binding domains (red box), and the LD domains (blue box). 


\section{FIGURE 3 | Continued}

The numbers indicate amino acid positions encoded within the C. caviae tarP gene. (B) Cos7 cells transfected with plasmids encoding full length TarP (TirM-TarP FL), progressive TarP deletion derivatives (TirM-TarP1-714 or TirM-TarP-1-639), or the TirM control were infected with $\Delta$ tir EPEC to induce clustering of the fusion protein. Transfected cells were identified by their ability to bind $\Delta$ tir EPEC. The white arrowheads indicate colocalization of vinculin (red) with $\Delta$ tir EPEC (blue). Vinculin was visualized with an anti-vinculin antibody. Bacteria were visualized by DAPI staining. Scale bars: $5 \mu \mathrm{m}$. Refer to Figures $\mathbf{4}, \mathbf{6 A}$ for schematic of TarP and its deletion derivatives. (C) Adhered EPEC able to recruit vinculin were enumerated for TirM, TirM-TarP-1-639, TirM-TarP1-714 and TirM-FL-TarP*, and data represented as box and whisker plot. Data compiled from three independent experiments. Plot shows range (statistical outliers excluded), first and third quartiles, and overall median (horizontal line). Diamonds show means. A range of 360-600 particles was counted. Insets show a magnification of a selected area of the cell. The asterisk and bars indicate significance difference between specific groups (One-way ANOVA, Tukey's post-hoc test, $P<0.00001$ ). NS, not significant.

incidence of vinculin recruitment. Scoring individual EPEC particles for colocalization with vinculin demonstrated that vinculin recruitment frequencies decreased 1.6 and 2.4-fold in cells expressing TirM- $\triangle$ VBS1 or TirM- $\triangle$ VBS1,2 respectively, relative to WT VBD (Figure 7C; $p<0.03$; ANOVA and TukeyKramer post-hoc test). Also, a VBD mutant derivative (VBS1 ${ }^{\text {mut }}$ $2-3$ ) in which the conserved hydrophobic residues of VBS1 were changed to serine was quantitatively and qualitatively similar to $\triangle$ VBS1. The data pointed to VBS1 accounting for the majority of VBD function.

A co-immunoprecipitation (co-IP) assay was conducted in parallel to the cell-based assays above. TarP-FL, the VBD and its derivatives were purified from transfected lysates by immunoprecipitation with an $\alpha-\mathrm{HA}$ tag monoclonal antibody. Vinculin co-precipitation was monitored in the anti-HA pulldown samples. Sample loading was adjusted for the amount of the HA signal pulled down to account for expression and immunoprecipitation levels. An asterisk indicated the band for each fusion protein. As shown in Figure 7D, TarP-FL and VBD interacted with vinculin. Because of the overwhelming strength of the vinculin signal in both TarP-FL and VBD, it was necessary to limit the exposure to a shorter time than the rest of the blot. The co-IP studies also confirmed the predominant role of VBS1 in vinculin binding, as VBS1 deletion or mutation diminished the levels of vinculin pulled down. Interestingly, the respective levels of pulldown by TarP-FL and VBD were considerably greater than those derivatives retaining VBS1. This is in contrast to the set of data obtained by microscopy. This could be due to the different experimental systems, where the cell-based approaches monitored vinculin recruitment at the plasma membrane, whereas co-IP did not distinguish between differently localized TarP-vinculin complexes, some of which may have been cytosolic. In addition, the cell-based assay may have additional host cell factors that influenced the recruitment and retention of vinculin.

\section{The LD and VBD Motifs Function Independently in Recruiting FAK and Vinculin, Respectively}

In the context of TarP, the VBD represents a further contribution to the growing list of functional domains that subvert the host actin cytoskeleton. One of these is the LD motif, which overlaps with the previously characterized TarP F-actin binding domain (FAB1). This motif mediated a FAK and Arp2/3 complexdependent actin remodeling (Thwaites et al., 2014). Its close proximity to VBD hinted at a potential interaction between the two domains. Using the EPEC system, the functions of VBD and
LD were investigated by expressing the C-terminal 241 amino acid residues of TarP spanning both motifs (TirM-LD-VBD) (Figure 8A). The recruitment of either Tyr397-phosphorylated FAK (pY397-FAK) or vinculin was monitored in parallel with TirM-LD and TirM-VBD (Figure 8B). We reasoned that an additive or synergistic interaction would result in the increased incidence of recruitment of pY397-FAK and vinculin to the clusters of TirM-LD-VBD at the plasma membrane. What we observed was that the incidences of recruitment of vinculin or pY397-FAK by TirM-VBD were similar to those of either the $\mathrm{LD}$ or the VBD domain alone (Figures $8 \mathrm{C}, \mathrm{D}$ ), indicating that vinculin recruitment by the VBD motif did not interfere with the recruitment of FAK by the LD domain, and vice versa. There was neither mutual inhibition nor additive/synergistic interactions.

The LD, VBD, and LD-VBD domains were evaluated for actin recruitment using the EPEC assay by staining the samples with phalloidin and DAPI to visualize filamentous actin and adhered EPEC bacteria on the cell surface, respectively (Figure 9A). Incidences of recruitment were monitored in a single-blind experiment, and data was expressed as a box and whisker plot (Figure 9B). TirM-LD-VBD was able to recruit actin at a frequency similar to that obtained for TirM-VBD. The value obtained for TirM-LD was consistent with our previous report (Thwaites et al., 2014). Based on the data, the FAK/LD and VBD/vinculin pathways do not exhibit an additive or synergistic interactions. It was not feasible to distinguish the relative contributions of each to the LD-VBD-mediated actin recruitment, and we could not exclude the possibility that signaling from the VBD inhibited that from the LD domain. We deem this unlikely, as FAK recruitment by the LD motif was not inhibited when VBD and vinculin were present.

\section{DISCUSSION}

Vinculin is exploited by a number of microbial pathogens as a means to productive infection. S. flexneri, Rickettsia, and enteropathogenic $E$. coli have all been reported to subvert vinculin functions directly through type III-secreted effectors ( $S$. flexneri and EPEC) (Freeman et al., 2000; Park et al., 2011b) or cell surface antigens (Rickettsia) (Park et al., 2011a). In each case, vinculin recruitment is regarded as a mechanism to induce localized actin remodeling. Here, we show that vinculin is a cellular target for the invasion-associated effector TarP, and is required for chlamydial entry. Our results reveal that Chlamydiae have engineered VBS that share a remarkable level of homology with the VBSs of talin. It is now clear that Chlamydia employ 
A

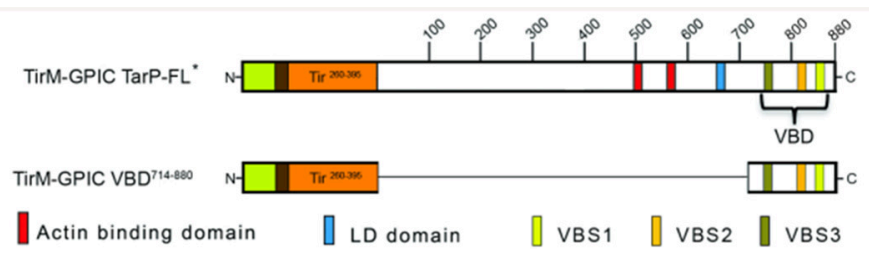

B
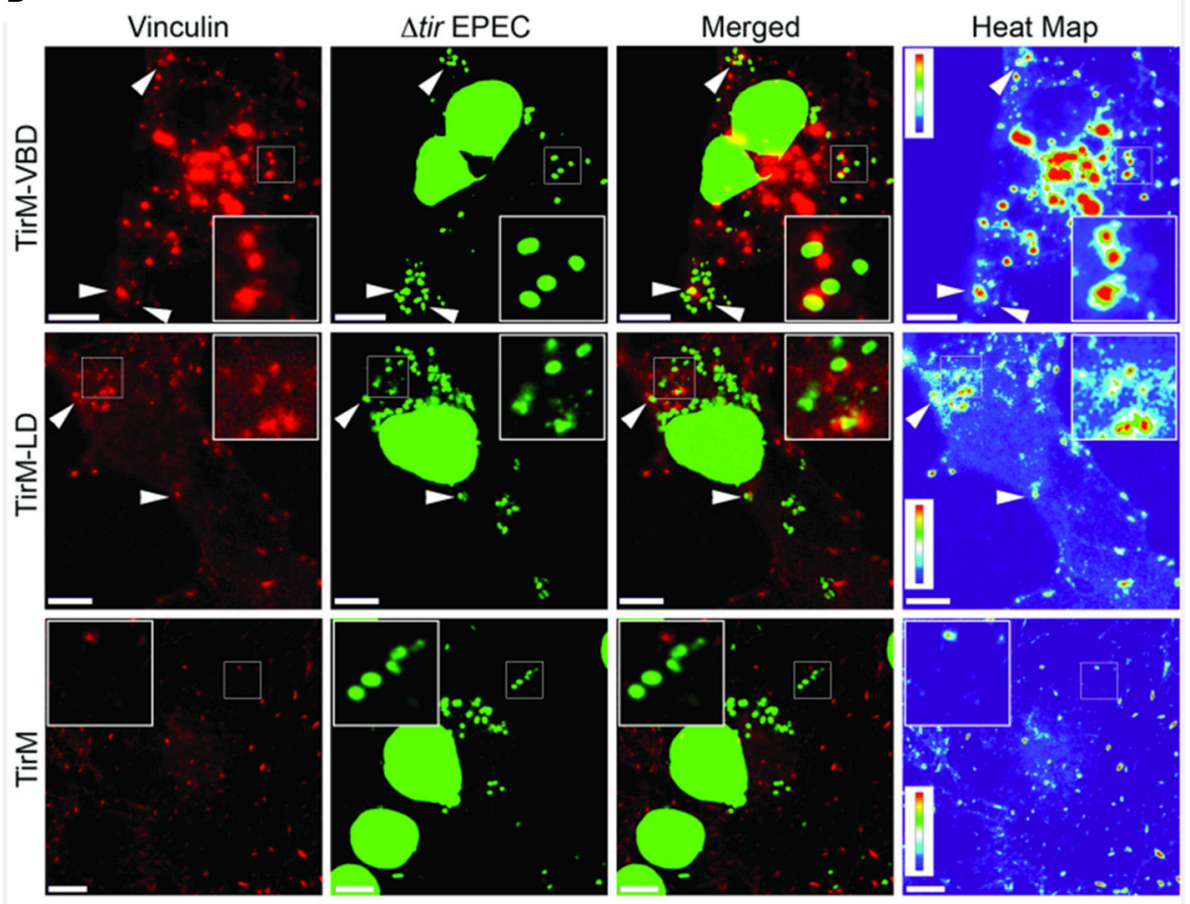

C

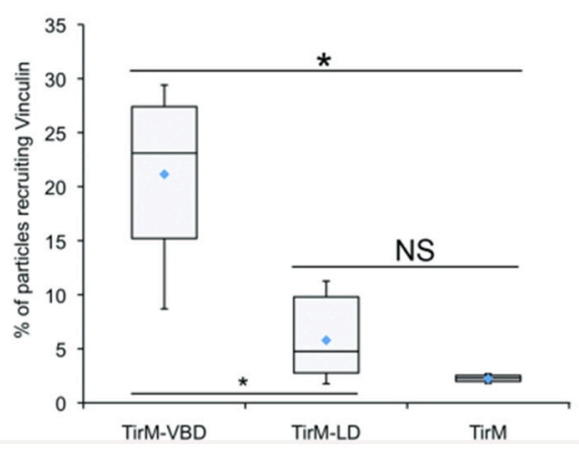

FIGURE 4 | The vinculin binding domain of TarP is functional and able to recruit vinculin. (A) Schematic of full length C. caviae TarP deleted of the proline rich domain (TarP FL ${ }^{*}$ ) and the TarP Vinculin Binding Domain (VBD; TirM-VBD) indicating the locations of the N-terminal membrane targeting sequence (yellow box), Ha-tag (brown box), TirM (amino acids 260-395; orange box), actin binding domains (red box), the LD domains (blue box), Vinculin Binding Site 3 (VBS; brown box), VBS2 (light orange box), and C-terminal VBS1 (yellow box). The numbers indicate amino acid positions encoded within the C. caviae TarP gene. *Denotes TarP derivative deleted for the PRD. (B) Cos7 cells transfected with plasmids encoding the VBD (TirM-VBD), the LD domain (TirM-LD), or the TirM control were infected with $\triangle$ tir EPEC to induce clustering of the fusion protein. Transfected cells were identified by their ability to bind $\Delta$ tir EPEC. Heat map of the vinculin channel highlights the intensity of vinculin recruited to sites of bacterial adherence. The white arrowheads indicate colocalization of vinculin (red) with $\Delta$ tir EPEC (false-colored green). Vinculin was visualized with an anti-vinculin antibody. Bacteria were visualized by DAPI staining. Scale bars: $10 \mu \mathrm{m}$. Heat map representation (red, high; violet, low) was generated using imageJ. (C) Adhered EPEC able to recruit vinculin were enumerated for TirM, TirM-LD and TirM_VBD, and data represented as box and whisker plot. Data compiled from three independent experiments. Plot shows range (statistical outliers excluded), first and third quartiles, and overall median (horizontal line). Diamonds show means. A range of 310-680 particles was counted. Insets show a magnification of a selected area of the cell. The asterisk and bars indicate significance difference between specific groups (One-way ANOVA, Tukey's post-hoc test, $P<0.00001$ ). NS, not significant. 


\section{A}

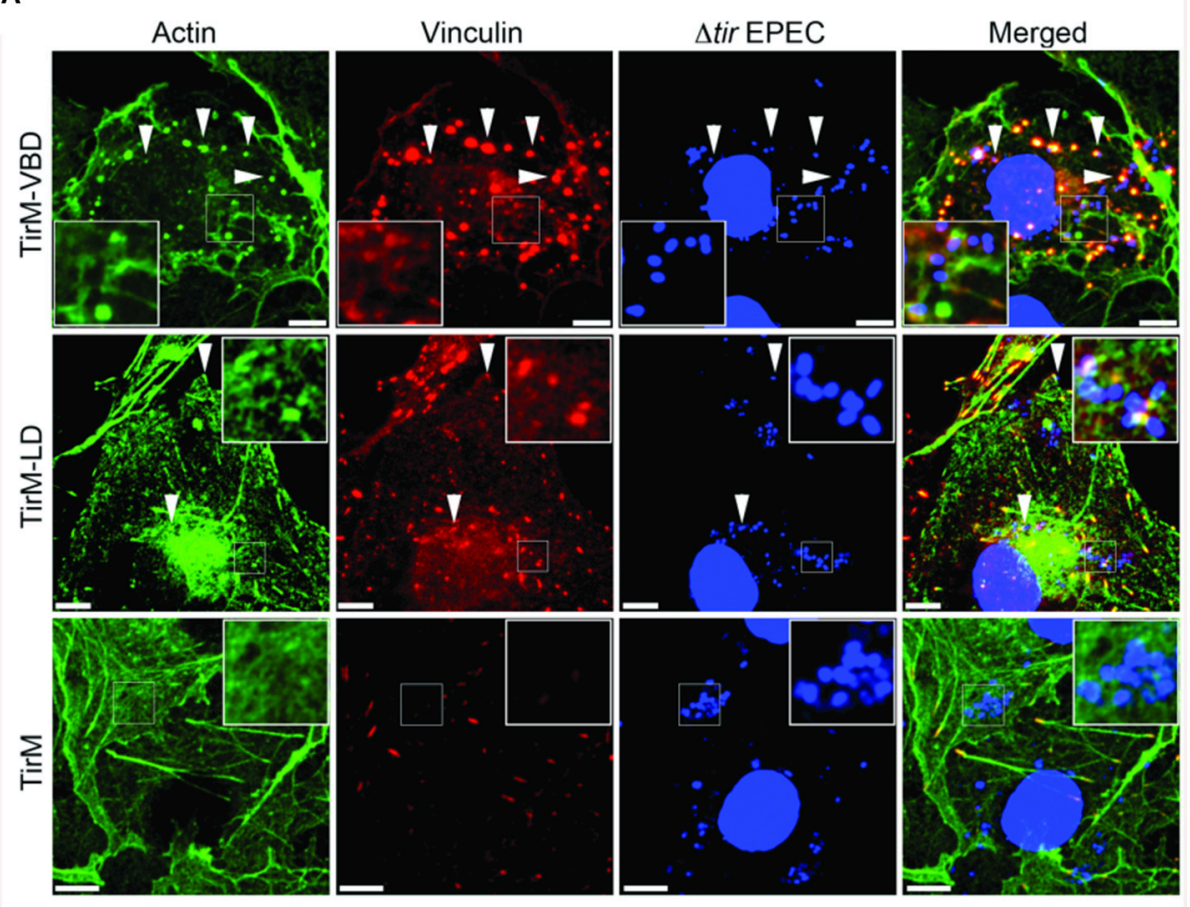

B

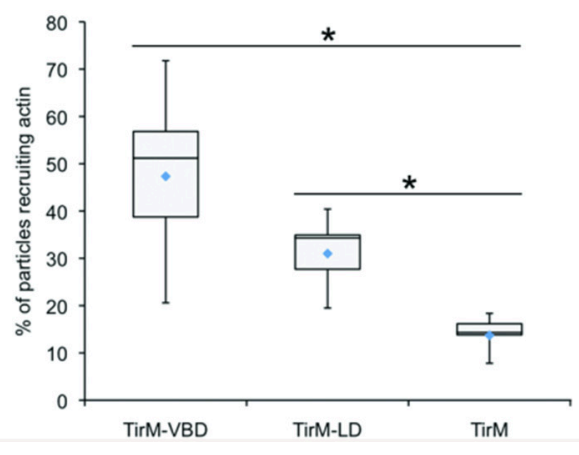

FIGURE 5 | The vinculin binding domain mediates the recruitment of both vinculin and actin. (A) Cos7 cells transfected with the plasmid encoding the TarP Vinculin Binding Domain (TirM-VBD), the LD domain (TirM-LD), or the control vector TirM were infected with $\triangle$ tir EPEC to induce clustering of the proteins. Transfected cells were identified by staining for the HA-tag present in the fusion proteins. The white arrowheads indicate colocalization of actin and vinculin with adhered $\triangle$ tir EPEC. Filamentous actin (green) and vinculin (red) were visualized with phalloidin or an anti-vinculin antibody, respectively. Bacteria (blue) were visualized by DAPI. Scale bars: $10 \mu \mathrm{m}$. (B) Adhered EPEC able to recruit actin were enumerated and data represented as box and whisker plot. Data compiled from three independent experiments. Plot shows range, first and third quartiles, and overall median (horizontal line). Diamonds indicate means. A range of 600-730 particles was counted. Insets show a magnification of a selected area of the cell. The asterisk and bars indicate significance difference between specific groups (One-way ANOVA, Tukey's post-hoc test, $P<0.00001$ ).

a number of strategies to hijack actin polymerization, with evidence pointing toward mimicry as the prevailing theme.

An important component of focal adhesion structures, vinculin functions to anchor the actin cytoskeleton to the plasma membrane via the b1 cytoplasmic domain of integrins. Binding of the tail domain to F-actin requires that vinculin adopts an activated "open conformation." Recently, the VBSs of talin have been reported to trigger vinculin activation by binding to its head domain and displacing interactions with tail domain (Bois et al., 2006). The binding of the Sca4 or IpaA VBSs to the head domain can displace the intramolecular interactions with the tail domain to facilitate vinculin activation and, ultimately, actin recruitment (Tran Van Nhieu et al., 1997; Hamiaux et al., 2006; Park et al., 2011a,b). Importantly, this scenario bypasses the "pre-activation" steps described for talin-mediated vinculin activation (Fillingham et al., 2005; del Rio et al., 2009; Grashoff et al., 2010), allowing these effectors to efficiently sequester this Factin binding protein for pathogenic purposes. Interestingly, the recruitment of vinculin observed during Chlamydia infection was due to a C-terminal VBD within TarP. Chlamydia TarP orthologs 


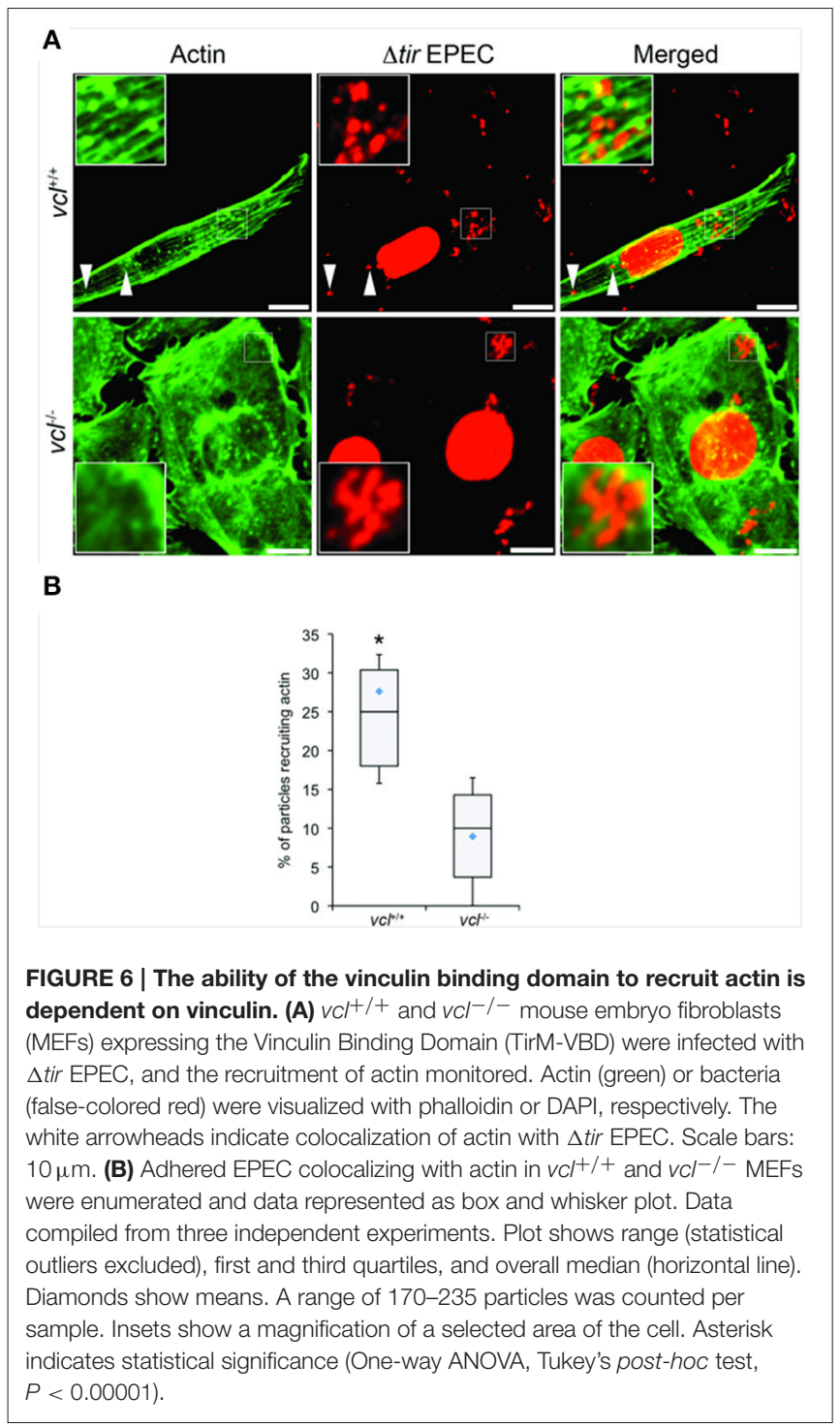

harbor variable numbers of VBS motifs that together comprise the VBD. Like IpaA, the VBD of C. caviae TarP consists of three VBS motifs and functions to recruit actin in a vinculin-dependent fashion. The high local concentration of these VBS motifs would ensure a tight association with vinculin. Indeed, such may be the case for S. flexneri. Izard et al. proposed that IpaA alters vinculin function through its high-affinity interaction between its VBS1 and the vinculin head domain (Izard et al., 2006). It was proposed that this interaction bypasses the force generation requirement to mechanically stretch and activate vinculin.

Interestingly, TarP harbors three VBSs with variations in amino acid residues in the hydrophobic face of the amphipathic $\alpha$-helices that likely manifest as differences in the strength of interaction with vinculin. Izard et al. reported that there are at least two residues of the IpaA-VBS1 consensus, Tyr-2 and Ala4 , which contribute to very high-affinity interactions with the hydrophobic interface with vinculin (Izard et al., 2006). For the TarP VBS motifs, it is clear that there is a perfect conservation of the alanine residue at position 4 and considerable variation at position 2. The three VBSs of IpaA appear to have different roles in Shigella entry, which has been traced to differences in affinity for vinculin (reviewed in Carayol and Tran Van Nhieu, 2013). The most carboxy-terminal VBS1 promotes vinculin activation by functioning as a supermimic of talin. Coincidentally, the most C-terminal VBS of TarP (VBS1), which most closely resembled the IpaA VBS1 (Figure S2), demonstrated the highest level of vinculin recruitment and was critical for vinculin interaction in pulldown experiments. Thus, it appears that the configuration of TarP VBSs reflects that found in IpaA. It would be of interest to investigate in greater detail the functions of TarP VBS2 and VBS3, which may include stabilization and maturation of the protein complex to maintain vinculin activation and actin binding.

The dependence of actin recruitment on vinculin contrasts with the findings of Jiwani et al. which showed a direct F-actin binding activity of TarP via the newly discovered FAB1 and FAB2 domains, the latter overlapping with C. caviae GPIC VBS3 (Jiwani et al., 2013). It was clear both from image acquisition and quantification of the level of actin recruitment that the VBD did not efficiently recruit actin to the plasma membrane in $\mathrm{vcl}^{-/-}$cells. If the TarP VBD had F-actin binding activity in the context of cultured cells, actin should have been present. We showed in a separate study that FAB1, which overlaps with the LD domain, recruits actin in a manner dependent on FAK (Thwaites et al., 2014). This discrepancy could be explained by the different experimental systems used. Whereas, Jiwani et al. used purified TarP derivatives to interrogate F-actin binding in vitro, we relied on ectopic expression in cells and monitored the recruitment of endogenous vinculin and actin. It is important to emphasize that, whilst interaction with vinculin may be important in invasion, we cannot discount the possibility of post-invasion functions for FAB1 and FAB2.

Interestingly, the duration of vinculin localization to the sites of invasion was longer than that of actin. At $120 \mathrm{~min}$, which is the latest time point studied, vinculin remained present. This is in contrast to the transient recruitment of actin, which we reported to be less than $5 \mathrm{~min}$ (Carabeo et al., 2002). It is possible that after the termination of signaling, vinculin remained associated with TarP. It is intriguing to speculate that this persistent interaction is significant in post-invasion stages of infection.

From a mechanistic point of view, it remains unclear how vinculin mediates chlamydia invasion. Our observations that FAK (Thwaites et al., 2014), vinculin (this study), talin, and paxillin (unpublished observations) were recruited to the sites of chlamydia invasion imply the formation of pseudo-focal adhesion structures that can organize the actin network to enable bacterial entry. A similar model has been proposed for Shigella (Tran Van Nhieu et al., 1997; Park et al., 2011b). This would imply that Chlamydia invasion is regulated to some extent by mechanisms similar to those that determine focal adhesion dynamics. Indeed, siRNA screens for host molecules necessary for Chlamydia infection have identified components of focal adhesion signaling, including DOCK180 (Elwell et al., 2008; Gurumurthy et al., 2010). Furthermore, the EB-associated type III effector TepP has been reported to interact with CrkI-II, which has a well-characterized role in FAK signaling (Zouq 
A

TirM-TarP-FL"

C

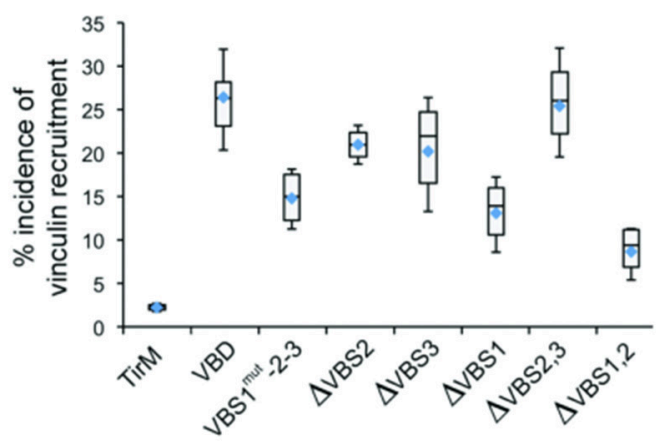

D

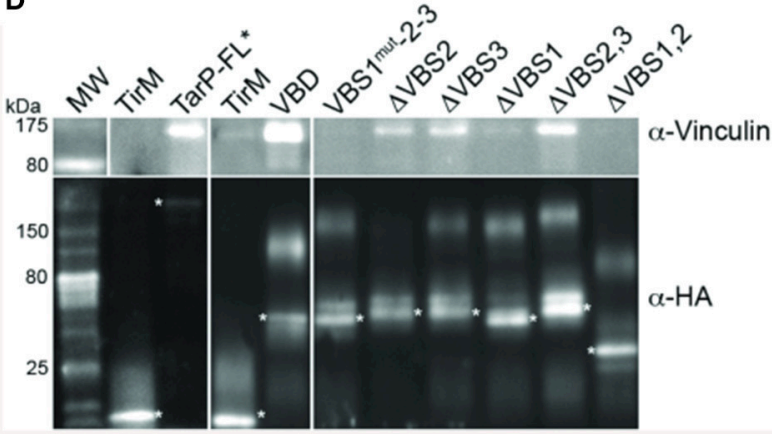

B
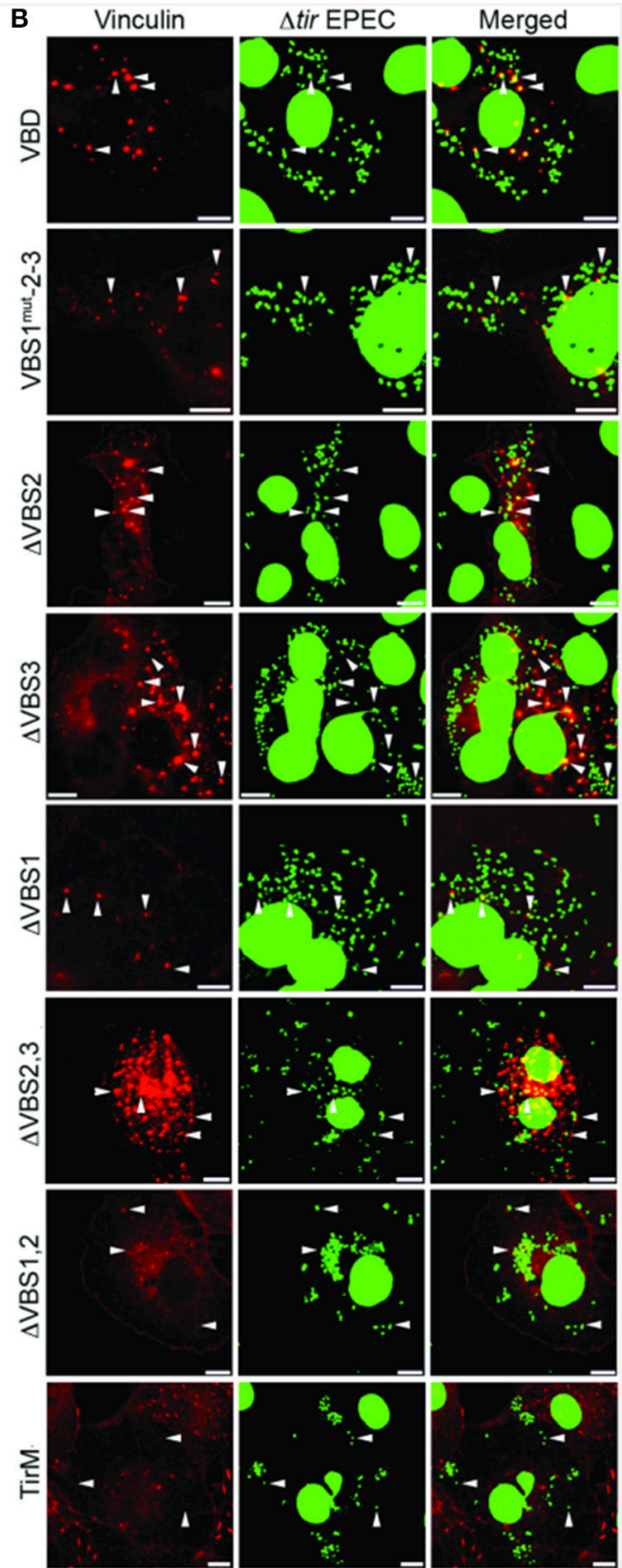

FIGURE 7 | Functional evaluation of the individual VBS motif revealed the importance of VBS1. (A) Schematic of Vinculin Binding Domain (TirM-VBD) derivatives designed for use with the EPEC-based assay. Indicated are the locations of the N-terminal membrane targeting sequence (MTS; cyan box), Ha-tag (red box), TirM (amino acids 260-395; purple box), proline rich domain (green box), actin binding domains (red box), the LD domains (blue box), Vinculin Binding Site 3 (VBS; brown box), VBS2 (orange box), and C-terminal VBS1 (yellow box). $\Delta$ indicates amino acids deleted in mutant TarP proteins, and the numbers indicate amino acid positions encoded within the C. caviae TarP gene. Yellow box with black stripes indicates a mutated VBS derivative. (B) Cos7 cells transfected with plasmids encoding the VBD (TirM-VBD), a VBD mutant derivative in which the critical leucines of VBS1 were converted to serines [TirM-VBS1(mut)-2-3], the VBD deleted for VBS1 (TirM- $\triangle$ VBS1), the VBD deleted for VBS2 (TirM- $\triangle$ VBS2), the VBD deleted for VBS3 (TirM- $\Delta$ VBS3), VBS1 or VBS3 were infected with $\triangle$ tir EPEC to induce clustering of the fusion protein. Transfected cells were identified by their ability to bind $\Delta$ tir EPEC. The white arrowheads indicate colocalization of Vinculin (red) with $\triangle$ tir EPEC (false-colored green). Vinculin was visualized with an anti-Vinculin antibody. Bacteria were visualized by DAPI staining. Scale bars: $10 \mu \mathrm{m}$. (C) Adhered EPEC able to recruit Vinculin were enumerated for VBD, VBS1(mut)-2-3, $\triangle$ VBS1, $\triangle$ VBS2, $\triangle$ VBS3, VBS1, or VBS3, and data represented as box and whisker plot. Data compiled from three independent experiments. The graph shows range (statistical outliers excluded), first and third quartiles, and overall median (horizontal line). Diamonds indicate means. A range of 200-550 particles was counted. The asterisk and bars indicate significant difference between specific groups (One-way ANOVA, Tukey's post-hoc test, $P<0.05)$. (D) The interaction of vinculin with the VBSs was evaluated by co-immunoprecipitation of HA-tagged VBD derivatives along with the full-length TarP and the negative control TirM at $32 \mathrm{~h}$ post-transfection. The co-precipitated vinculin protein and the recombinant TarP and/or VBD constructs were visualized by western blot using antibodies to vinculin and the HA-tag, respectively. The HA-tagged proteins are indicated by asterisks. Note that the loss of VBS1 either through deletion or mutation consistently led to the loss of interaction with vinculin. 
A

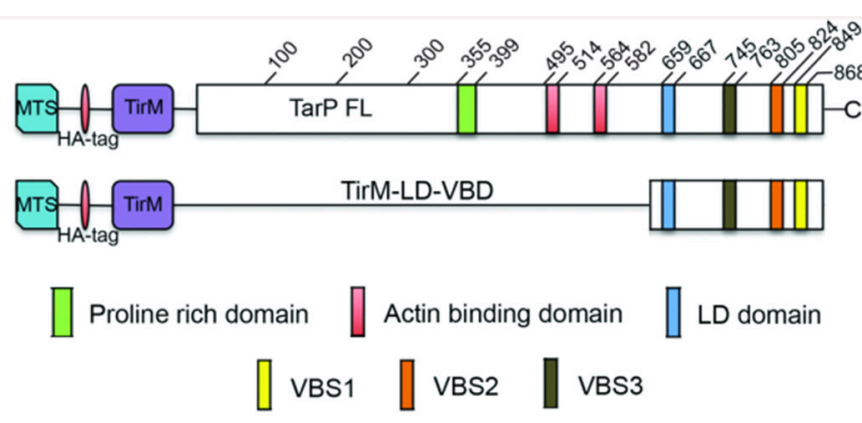

B
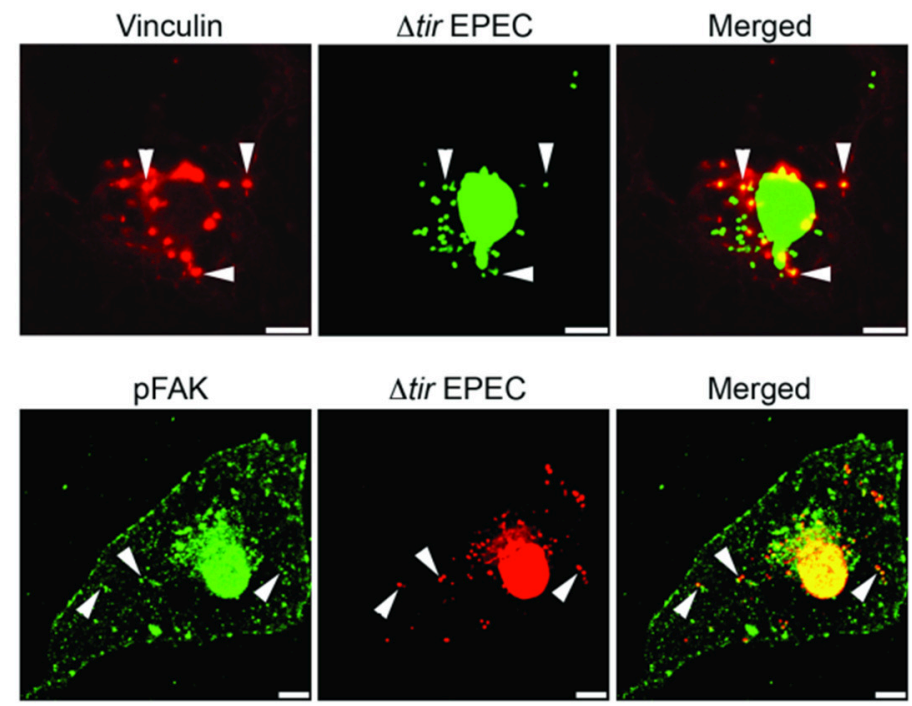

C

D
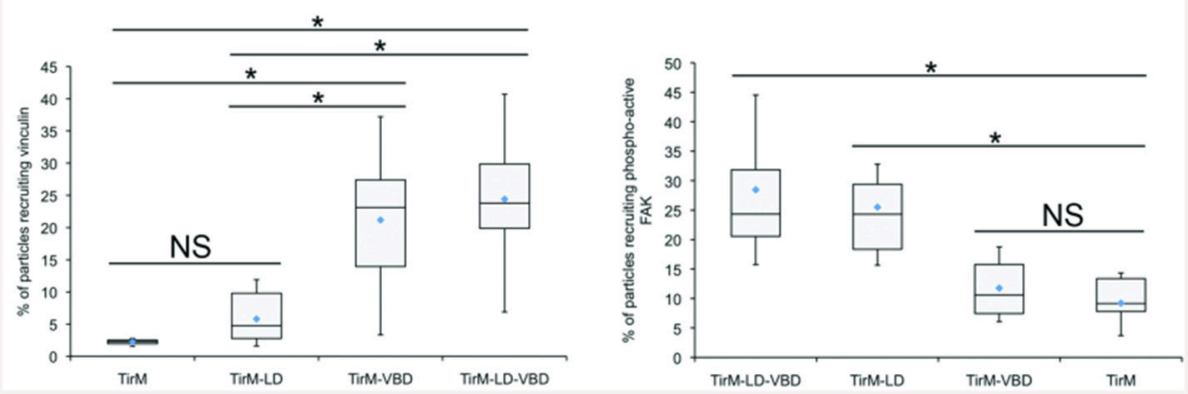

FIGURE 8 | The LD-VBD protein is functional and able to recruit phospho-active or vinculin. (A) Schematic of full length TarP (TarP-FL) or its derivative, the LD-VBD, which represents a combination of the TarP LD domain and the TarP Vinculin Binding Domain (VBD). Please see Figure 4 legend for details. (A,B) Cos7 cells transfected with the plasmid encoding LD-VBD were infected with $\triangle$ tir EPEC to induce clustering of the proteins. Transfected cells were identified by the capacity to facilitate bacteria attachment. The white arrowheads indicate colocalization of phospho-active FAK (pFAK) or vinculin with adhered $\triangle$ tir EPEC. pFAK (green) and vinculin (red) were visualized with an anti-FAK (phospho Y397) or an anti-vinculin antibody, respectively. Bacteria [false-colored green (vinculin) or red (pFAK)] were visualized by DAPI. Scale bars: $10 \mu \mathrm{m}$. (C,D) Adhered EPEC able to recruit vinculin (C) or pFAK (D) were enumerated and data represented as box and whisker plot. Data compiled from two independent experiments. Plot shows range (statistical outliers excluded), first and third quartiles, and overall median (horizontal line). Diamonds show means. Roughly 200 particles were counted for both conditions. The asterisk and bars indicate significance difference between specific groups (One-way ANOVA, Tukey's post-hoc test, $P<0.00001$ ). NS; not significant. For comparison, previously quantified data for the LD domain alone or the VBD domain alone with pFAK or vinculin was included.

et al., 2009; Chen et al., 2014) further supporting a pseudo-focal adhesion signaling model. The relatively detailed knowledge of focal adhesion signaling may serve as a good framework for the characterization of FAK- and vinculin-dependent functions of
TarP. An intriguing model implicates TarP in a role similar to that of integrin molecules, serving as a molecular scaffold to which focal adhesion-associated proteins bind and initiate signaling to and interaction with the host actin cytoskeleton. 


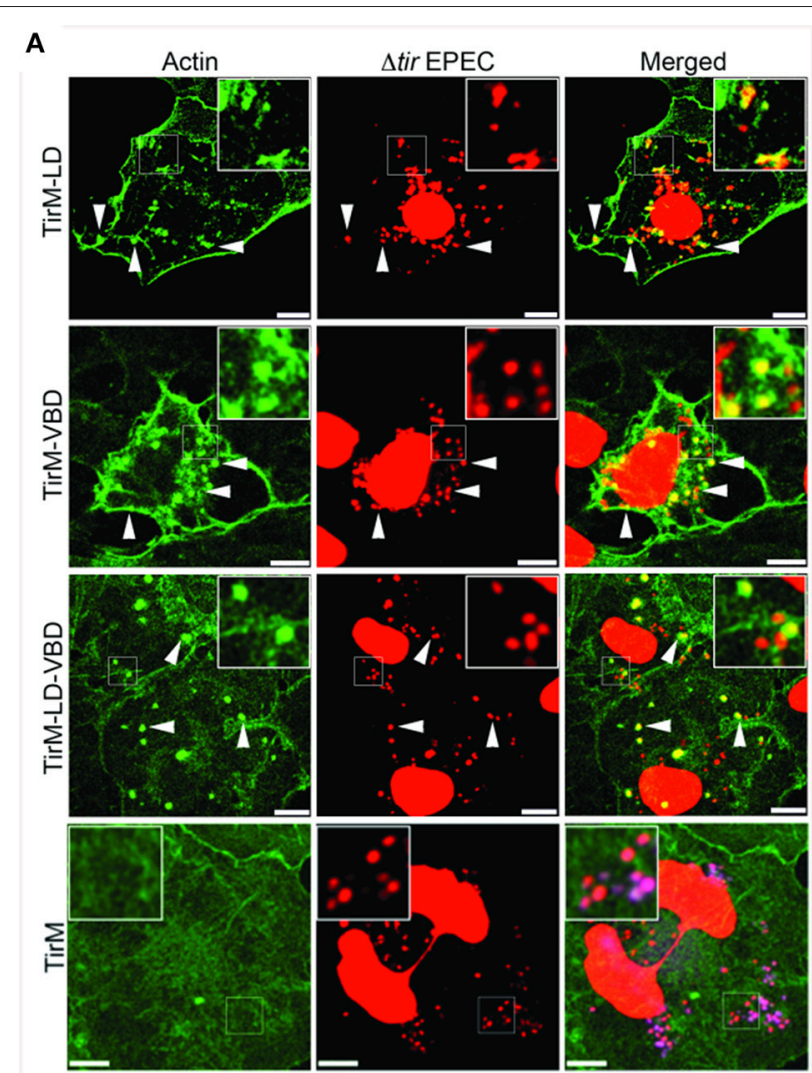

B

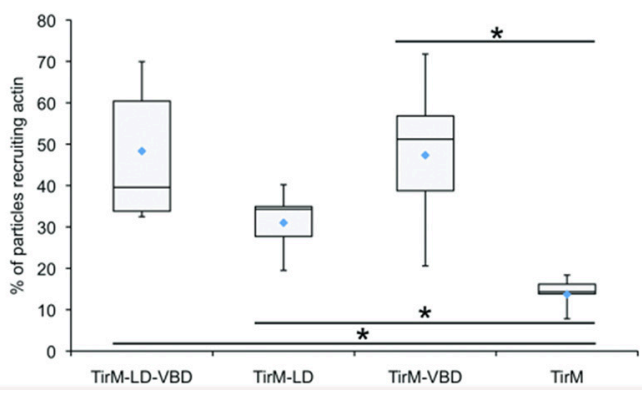

FIGURE 9 | The LD-VBD induces robust actin recruitment. (A) Cos7 cells transfected with the plasmid encoding LD-VBD were infected with $\triangle$ tir EPEC to induce clustering of the proteins. Transfected cells were identified by the capacity to facilitate bacteria attachment. The white arrowheads indicate colocalization of actin with adhered $\triangle$ tir EPEC. Actin (green) and bacteria (false-colored red) were visualized with phalloidin or DAPI, respectively. Scale bars: $10 \mu \mathrm{m}$. (B) Adhered EPEC able to recruit actin were enumerated and data represented as box and whisker plot. Data compiled from three independent experiments. Plot shows range (statistical outliers excluded), first and third quartiles, and overall median (horizontal line). Diamonds indicate means. A range of 300-680 particles were counted. The asterisk and bars indicate significance difference between specific groups (One-way ANOVA, Tukey's post-hoc test, $P<0.00001)$. For comparison, previously quantified data for the LD domain alone or the VBD domain alone actin was included.

Here, we addressed the molecular basis of vinculin recruitment at the adhesion sites, its role in actin recruitment, and in the uptake of Chlamydia EBs. During the course of this study, we also investigated the potential functional interaction between the LD and VBD motifs. While the LD and VBD motifs did not exhibit signaling cooperation, it remains possible that these domains function with other motifs, such as the phosphodomain of $C$. trachomatis, and the actin-binding domains or with pathways involving host cell receptors. Multiple pathways of invasion have been reported for Chlamydia. When one pathway is ablated, there was a consistent lack of complete compensation, i.e., other endogenous pathways that remained functional did not restore fully invasion efficiencies, indicating that the invasion pathways available to Chlamydia are non-redundant. It is intriguing to speculate that this may relate to cellular/tissue tropism in vivo, such as interaction of the pathogen with non-phagocytic and phagocytic cells, or columnar vs. stratified squamous epithelia. In addition, while these domains have been shown to function individually in vitro, it is a very intriguing possibility that in different contexts, they may also exhibit cooperative interactions to ensure an efficient actin remodeling to facilitate Chlamydia invasion.

\section{AUTHOR CONTRIBUTIONS}

TT, AP, TP, and RC conceived and designed the experiments. TT, AP, and TP performed the experiments and collected the data. TT, AP, TP, and RC analyzed the data. TT, AP, TP, and RC prepared the figures and wrote the manuscript.

\section{ACKNOWLEDGMENTS}

The authors would like to acknowledge Drs. Alex Wong and Keith S. Robinson (Imperial College London) for helpful suggestions and Dr. Wolfgang Ziegler (Hannover Medical School) and Prof. Gad Frankel (Imperial College London) for their kind gifts of reagents and bacterial strains. This research was supported by the Medical Research Council (G0900213) and the National Health Institutes (AI065545) to RC. TT was supported by a Medical Research Council studentship. AP is a recipient of a predoctoral fellowship from the Fundação para a Ciência e a Tecnologia of the Portuguese government.

\section{SUPPLEMENTARY MATERIAL}

The Supplementary Material for this article can be found online at: http://journal.frontiersin.org/article/10.3389/fcimb. 2015.00088

Figure S1 | Chlamydia recruits vinculin to sites of infection. C. caviae (GPIC) EBs were visualized with DAPI were added to Cos7 cells and the infection allowed to proceed for $0,10,30,60,90$, or 120 min as described in the Materials and Methods Section. The samples were stained with an anti-vinculin antibody and DAPI to visualize the bacteria. C. caviae EBs (green); vinculin (red). Scale bars: $5 \mu \mathrm{m}$. White arrowheads indicate vinculin/GPIC EB colocalization.

\section{Figure S2 | The VBS motifs of TarP adopt an amphipathic a-helical} structure. (A) Schematic of C. caviae GPIC TarP is shown indicating the positions and nomenclature of the VBS motifs. (B) ClustalW sequence alignment of the GPIC VBS motifs, the mutant derivative of VBS1 used in the context of TirM-VBS1(mut)-2-3 construct, and the C-terminal VBS1 from the Shigella flexneri effector IpaA. Identical amino acids within each alignment are in red. Similar 
residues are in blue. (C) Schiffer-Edmundson helical wheel diagrams depicting the 19 amino acids of VBS1, VBS2, VBS3, mutant VBS1, and IpaA VBS1 created with DNAstar program demonstrating the possible amphipathic $\alpha$-helical conformation. Helical wheel projects the arrangement of amino acids, and residue numbers are counted from the amino terminus. Amino acids are represented by the single letter code and are colored based on their chemical properties. Hydrophobic (I, L, V, M: black; A, G, P: Green), hydrophilic (S, T: yellow; Y: blue; N: pink), and charged (D, E: red; K, R, H: light blue). The lines indicate bonds between amino acids. The arrow indicates the direction of the calculated hydrophobic moment, which is quantitative measure of amphipathicity.

\section{REFERENCES}

Balañá, M. E., Niedergang, F., Subtil, A., Alcover, A., Chavrier, P., and Dautry-Varsat, A. (2005). ARF6 GTPase controls bacterial invasion by actin remodelling. J. Cell Sci. 118, 2201-2210. doi: 10.1242/jcs.02351

Bois, P. R., O’Hara, B. P., Nietlispach, D., Kirkpatrick, J., and Izard, T. (2006). The vinculin binding sites of talin and alpha-actinin are sufficient to activate vinculin. J. Biol. Chem. 281, 7228-7236. doi: 10.1074/jbc.M510397200

Caldwell, H. D., Kromhout, J., and Schachter, J. (1981). Purification and partial characterization of the major outer membrane protein of Chlamydia trachomatis. Infect. Immun. 31, 1161-1176.

Campellone, K. G., Rankin, S., Pawson, T., Kirschner, M. W., Tipper, D. J., and Leong, J. M. (2004). Clustering of Nck by a 12-residue Tir phosphopeptide is sufficient to trigger localized actin assembly. J. Cell Biol. 164, 407-416. doi: $10.1083 /$ jcb. 200306032

Carabeo, R. (2011). Bacterial subversion of host actin dynamics at the plasma membrane. Cell Microbiol. 13, 1460-1469. doi: 10.1111/j.14625822.2011.01651.x

Carabeo, R. A., Grieshaber, S. S., Fischer, E., and Hackstadt, T. (2002). Chlamydia trachomatis induces remodeling of the actin cytoskeleton during attachment and entry into HeLa cells. Infect. Immun. 70, 3793-3803. doi: 10.1128/IAI.70.7.3793-3803.2002

Carayol, N., and Tran Van Nhieu, G. (2013). Tips and tricks about Shigella invasion of epithelial cells. Curr. Opin. Microbiol. 16, 32-37. doi: 10.1016/j.mib.2012.11.010

Case, L. B., Baird, M. A., Shtengel, G., Campbell, S. L., Hess, H. F., Davidson, M. W., et al. (2015). Molecular mechanism of vinculin activation and nanoscale spatial organization in focal adhesions. Nat. Cell Biol. 17, 880-892. doi: $10.1038 /$ ncb3180

Chen, Y. S., Bastidas, R. J., Saka, H. A., Carpenter, V. K., Richards, K. L., Plano, G. V., et al. (2014). The Chlamydia trachomatis type III secretion chaperone Slc1 engages multiple early effectors, including TepP, a tyrosinephosphorylated protein required for the recruitment of CrkI-II to nascent inclusions and innate immune signaling. PLoS Pathog. 10:e1003954. doi: 10.1371/journal.ppat.1003954

Clifton, D. R., Dooley, C. A., Grieshaber, S. S., Carabeo, R. A., Fields, K. A., and Hackstadt, T. (2005). Tyrosine phosphorylation of the chlamydial effector protein Tarp is species specific and not required for recruitment of actin. Infect. Immun. 73, 3860-3868. doi: 10.1128/IAI.73.7.3860-3868.2005

Coombes, B. K., and Mahony, J. B. (2002). Identification of MEK- and phosphoinositide 3-kinase-dependent signalling as essential events during Chlamydia pneumoniae invasion of HEp2 cells. Cell Microbiol. 4, 447-460. doi: 10.1046/j.1462-5822.2002.00203.x

Critchley, D. R., Holt, M. R., Barry, S. T., Priddle, H., Hemmings, L., and Norman, J. (1999). Integrin-mediated cell adhesion: the cytoskeletal connection. Biochem. Soc. Symp. 65, 79-99.

del Rio, A., Perez-Jimenez, R., Liu, R., Roca-Cusachs, P., Fernandez, J. M., and Sheetz, M. P. (2009). Stretching single talin rod molecules activates vinculin binding. Science 323, 638-641. doi: 10.1126/science.1162912

DeMali, K. A., Barlow, C. A., and Burridge, K. (2002). Recruitment of the Arp2/3 complex to vinculin: coupling membrane protrusion to matrix adhesion. J. Cell Biol. 159, 881-891. doi: 10.1083/jcb.200206043

Elwell, C. A., Ceesay, A., Kim, J. H., Kalman, D., and Engel, J. N. (2008). RNA interference screen identifies Abl kinase and PDGFR signaling in Chlamydia trachomatis entry. PLoS Pathog. 4:e1000021. doi: 10.1371/journal.ppat. 1000021

Fillingham, I., Gingras, A. R., Papagrigoriou, E., Patel, B., Emsley, J., Critchley, D. R., et al. (2005). A vinculin binding domain from the talin rod unfolds to form a complex with the vinculin head. Structure 13, 65-74. doi: 10.1016/j.str.2004.11.006

Finlay, B. B., Ruschkowski, S., and Dedhar, S. (1991). Cytoskeletal rearrangements accompanying Salmonella entry into epithelial cells. J. Cell Sci. 99, 283-296.

Freeman, N. L., Zurawski, D. V., Chowrashi, P., Ayoob, J. C., Huang, L., Mittal, B., et al. (2000). Interaction of the enteropathogenic Escherichia coli protein, translocated intimin receptor (Tir), with focal adhesion proteins. Cell Motil. Cytoskeleton 47, 307-318. doi: 10.1002/1097-0169(200012)47:4<307::AIDCM5>3.0.CO;2-Q

Gingras, A. R., Ziegler, W. H., Frank, R., Barsukov, I. L., Roberts, G. C., Critchley, D. R., et al. (2005). Mapping and consensus sequence identification for multiple vinculin binding sites within the talin rod. J. Biol. Chem. 280, 37217-37224. doi: 10.1074/jbc.M508060200

Grashoff, C., Hoffman, B. D., Brenner, M. D., Zhou, R., Parsons, M., Yang, M. T., et al. (2010). Measuring mechanical tension across vinculin reveals regulation of focal adhesion dynamics. Nature 466, 263-266. doi: 10.1038/ nature 09198

Gurumurthy, R. K., Mäurer, A. P., Machuy, N., Hess, S., Pleissner, K. P., Schuchhardt, J., et al. (2010). A loss-of-function screen reveals Ras- and Rafindependent MEK-ERK signaling during Chlamydia trachomatis infection. Sci. Signal. 3, ra21. doi: 10.1126/scisignal.2000651

Hamiaux, C., van Eerde, A., Parsot, C., Broos, J., and Dijkstra, B. W. (2006). Structural mimicry for vinculin activation by IpaA, a virulence factor of Shigella flexneri. EMBO Rep. 7, 794-799. doi: 10.1038/sj.embor.7400753

Humphries, J. D., Wang, P., Streuli, C., Geiger, B., Humphries, M. J., and Ballestrem, C. (2007). Vinculin controls focal adhesion formation by direct interactions with talin and actin. J. Cell Biol. 179, 1043-1057. doi: $10.1083 /$ jcb. 200703036

Hüttelmaier, S., Mayboroda, O., Harbeck, B., Jarchau, T., Jockusch, B. M., and Rüdiger, M. (1998). The interaction of the cell-contact proteins VASP and vinculin is regulated by phosphatidylinositol-4,5-bisphosphate. Curr. Biol. 8, 479-488. doi: 10.1016/S0960-9822(98)70199-X

Izard, T., Tran Van Nhieu, G., and Bois, P. R. (2006). Shigella applies molecular mimicry to subvert vinculin and invade host cells. J. Cell Biol. 175, 465-475. doi: $10.1083 /$ jcb. 200605091

Jewett, T. J., Fischer, E. R., Mead, D. J., and Hackstadt, T. (2006). Chlamydial TARP is a bacterial nucleator of actin. Proc. Natl. Acad. Sci. U.S.A. 103, 15599-15604. doi: 10.1073/pnas.0603044103

Jiwani, S., Alvarado, S., Ohr, R. J., Romero, A., Nguyen, B., and Jewett, T. J. (2013). Chlamydia trachomatis Tarp harbors distinct $\mathrm{G}$ and $\mathrm{F}$ actin binding domains that bundle actin filaments. J. Bacteriol. 195, 708-716. doi: 10.1128/JB. 01768-12

Jiwani, S., Ohr, R. J., Fischer, E. R., Hackstadt, T., Alvarado, S., Romero, A., et al. (2012). Chlamydia trachomatis Tarp cooperates with the Arp $2 / 3$ complex to increase the rate of actin polymerization. Biochem. Biophys. Res. Commun. 420, 816-821. doi: 10.1016/j.bbrc.2012.03.080

Johnson, R. P., and Craig, S. W. (1995). F-actin binding site masked by the intramolecular association of vinculin head and tail domains. Nature 373, 261-264. doi: 10.1038/373261a0

Lane, B. J., Mutchler, C., Al Khodor, S., Grieshaber, S. S., and Carabeo, R. A. (2008). Chlamydial entry involves TARP binding of guanine nucleotide exchange factors. PLoS Pathog. 4:e1000014. doi: 10.1371/journal.ppat.1000014

Marg, S., Winkler, U., Sestu, M., Himmel, M., Schönherr, M., Bär, J., et al. (2010). The vinculin-DeltaIn20/21 mouse: characteristics of a constitutive, actin-binding deficient splice variant of vinculin. PLOS ONE 5:e11530. doi: 10.1371/journal.pone.0011530

Mehlitz, A., and Rudel, T. (2013). Modulation of host signaling and cellular responses by Chlamydia. Cell Commun. Signal. 11:90. doi: 10.1186/1478-811X$11-90$

Park, H., Lee, J. H., Gouin, E., Cossart, P., and Izard, T. (2011a). The rickettsia surface cell antigen 4 applies mimicry to bind to and activate vinculin. J. Biol. Chem. 286, 35096-35103. doi: 10.1074/jbc.M111.263855 
Park, H., Valencia-Gallardo, C., Sharff, A., Tran Van Nhieu, G., and Izard, T. (2011b). Novel vinculin binding site of the IpaA invasin of Shigella. J. Biol. Chem. 286, 23214-23221. doi: 10.1074/jbc.M110. 184283

Schneider, C. A., Rasband, W. S., and Eliceiri, K. W. (2012). NIH Image to ImageJ: 25 years of image analysis. Nat. Methods 9, 671-675. doi: 10.1038/ nmeth.2089

Subtil, A., Wyplosz, B., Balañá, M. E., and Dautry-Varsat, A. (2004). Analysis of Chlamydia caviae entry sites and involvement of Cdc42 and Rac activity. J. Cell Sci. 117, 3923-3933. doi: 10.1242/jcs.01247

Thwaites, T., Nogueira, A. T., Campeotto, I., Silva, A. P., Grieshaber, S. S., and Carabeo, R. A. (2014). The Chlamydia effector TarP mimics the mammalian leucine-aspartic acid motif of paxillin to subvert the focal adhesion kinase during invasion. J. Biol. Chem. 289, 30426-30442. doi: 10.1074/jbc.M114.604876

Tran Van Nhieu, G., Ben-Ze'ev, A., and Sansonetti, P. J. (1997). Modulation of bacterial entry into epithelial cells by association between vinculin and the Shigella IpaA invasin. EMBO J. 16, 2717-2729. doi: 10.1093/emboj/ 16.10.2717

Tran Van Nhieu, G., and Izard, T. (2007). Vinculin binding in its closed conformation by a helix addition mechanism. EMBO J. 26, 4588-4596.
Turner, C. E., Glenney, J. R. Jr., and Burridge, K. (1990). Paxillin: a new vinculinbinding protein present in focal adhesions. J. Cell Biol. 111, 1059-1068. doi: 10.1083/jcb.111.3.1059

Wong, A. R., Raymond, B., Collins, J. W., Crepin, V. F., and Frankel, G. (2012). The enteropathogenic E. coli effector EspH promotes actin pedestal formation and elongation via WASP-interacting protein (WIP). Cell Microbiol. 14, 1051-1070. doi: 10.1111/j.1462-5822.2012.01778.x

Zouq, N. K., Keeble, J. A., Lindsay, J., Valentijn, A. J., Zhang, L., Mills, D., et al. (2009). FAK engages multiple pathways to maintain survival of fibroblasts and epithelia: differential roles for paxillin and p130Cas. J. Cell Sci. 122, 357-367. doi: $10.1242 /$ jcs.030478

Conflict of Interest Statement: The authors declare that the research was conducted in the absence of any commercial or financial relationships that could be construed as a potential conflict of interest.

Copyright (c) 2015 Thwaites, Pedrosa, Peacock and Carabeo. This is an open-access article distributed under the terms of the Creative Commons Attribution License (CC $B Y)$. The use, distribution or reproduction in other forums is permitted, provided the original author(s) or licensor are credited and that the original publication in this journal is cited, in accordance with accepted academic practice. No use, distribution or reproduction is permitted which does not comply with these terms. 